\title{
Hertzian Dipole Radiation over Isotropic Magnetodielectric Substrates
}

\author{
by Gregory A Talalai
}

ARL-TR-7237

March 2015 


\section{NOTICES}

\section{Disclaimers}

The findings in this report are not to be construed as an official Department of the Army position unless so designated by other authorized documents.

Citation of manufacturer's or trade names does not constitute an official endorsement or approval of the use thereof.

Destroy this report when it is no longer needed. Do not return it to the originator. 


\title{
Army Research Laboratory
}

Adelphi, MD 20783-1138

ARL-TR-7237

March 2015

\section{Hertzian Dipole Radiation over Isotropic Magnetodielectric Substrates}

\author{
Gregory A Talalai
}

Sensors and Electron Devices Directorate, ARL 
Public reporting burden for this collection of information is estimated to average 1 hour per response, including the time for reviewing instructions, searching existing data sources, gathering and maintaining the data needed, and completing and reviewing the collection information. Send comments regarding this burden estimate or any other aspect of this collection of information, including suggestions for reducing the burden, to Department of Defense, Washington Headquarters Services, Directorate for Information Operations and Reports (0704-0188), 1215 Jefferson Davis Highway, Suite 1204, Arlington, VA 22202-4302. Respondents should be aware that notwithstanding any other provision of law, no person shall be subject to any penalty for failing to comply with a collection of information if it does not display a currently valid OMB control number.

PLEASE DO NOT RETURN YOUR FORM TO THE ABOVE ADDRESS.

\begin{tabular}{|c|c|c|}
\hline $\begin{array}{l}\text { 1. REPORT DATE }(D D-M M-Y Y Y Y) \\
\text { March } 2015\end{array}$ & $\begin{array}{l}\text { 2. REPORT TYPE } \\
\text { Final }\end{array}$ & 3. DATES COVERED (From - To) \\
\hline \multirow{3}{*}{\multicolumn{2}{|c|}{$\begin{array}{l}\text { 4. TITLE AND SUBTITLE } \\
\text { Hertzian Dipole Radiation over Isotropic Magnetodielectri }\end{array}$}} & 5a. CONTRACT NUMBER \\
\hline & & 5b. GRANT NUMBER \\
\hline & & 5c. PROGRAM ELEMENT NUMBER \\
\hline \multirow{3}{*}{\multicolumn{2}{|c|}{$\begin{array}{l}\text { 6. AUTHOR(S) } \\
\text { Gregory A Talalai }\end{array}$}} & 5d. PROJECT NUMBER \\
\hline & & 5e. TASK NUMBER \\
\hline & & 5f. WORK UNIT NUMBER \\
\hline \multicolumn{2}{|c|}{$\begin{array}{l}\text { US Army Research Laboratory } \\
\text { ATTN: RDRL-SER-M } \\
\text { 2800 Powder Mill Road } \\
\text { Adelphi, MD 20783-1138 }\end{array}$} & $\begin{array}{l}\text { 8. PERFORMING ORGANIZATION } \\
\text { REPORT NUMBER } \\
\text { ARL-TR-7237 }\end{array}$ \\
\hline \multicolumn{2}{|c|}{ 9. SPONSORING/MONITORING AGENCY NAME(S) AND ADDRESS(ES) } & 10. SPONSOR/MONITOR'S ACRONYM(S) \\
\hline \multicolumn{2}{|c|}{$\begin{array}{l}\text { US ARMY CERDEC S\&TCD } \\
\text { Bldg 6010, Frankford Street, Room } 404 \\
\text { APG, MD } 21005\end{array}$} & $\begin{array}{l}\text { 11. SPONSOR/MONITOR'S REPORT } \\
\text { NUMBER(S) }\end{array}$ \\
\hline
\end{tabular}

APG, MD 21005

12. DISTRIBUTION/AVAILABILITY STATEMENT

Approved for public release; distribution unlimited.

13. SUPPLEMENTARY NOTES

\section{ABSTRACT}

This report investigates dipole antennas printed on grounded magnetodielectric substrates. The interest in magnetodielectric substrates stems from the expectation that new magnetic materials may revolutionize the engineering of thin planar antennas. Since these materials often require complicated constitutive equations to describe their properties rigorously, the solution of intricate boundary value problems has become important to understanding the associated radiating structures. In this report, the magnetodielectric is modeled as a simple material with scalar constitutive parameters, an approximation that is likely not satisfactory. However, with such an approximation, our problem reduces to a very well-known boundary value problem in applied electromagnetics work. Despite the extensive amount of existing literature, no completely self-contained treatment of the mathematical boundary value problem itself was found. Consequently, the missing mathematical details are provided in this report with the intent to be useful to future research of magnetic materials described by more realistic constitutive equations. Furthermore, some novel formulas are given as they relate to the square roots appearing in the theory of complex integration. The directivity, radiation resistance, and efficiency are derived, and some results are plotted, from which we observe complicated dependencies on material properties and substrate thickness.

\section{SUBJECT TERMS}

Magnetodielectrics, planar antennas, boundary value problems, contour integration, branch cuts, Sommerfeld integrals

\begin{tabular}{|l|l|l|c|c|l|}
\hline \multicolumn{3}{|l|}{ 16. SECURITY CLASSIFICATION OF: } & $\begin{array}{c}\text { 17. LIMITATION } \\
\text { OF } \\
\text { ABSTRACT }\end{array}$ & $\begin{array}{c}\text { 18. NUMBER } \\
\text { OF } \\
\text { PAGES }\end{array}$ & $\begin{array}{l}\text { 19a. NAME OF RESPONSIBLE PERSON } \\
\text { Gregory A Talalai }\end{array}$ \\
\cline { 1 - 2 } $\begin{array}{l}\text { a. REPORT } \\
\text { Unclassified }\end{array}$ & $\begin{array}{c}\text { b. ABSTRACT } \\
\text { Unclassified }\end{array}$ & $\begin{array}{c}\text { c. THIS PAGE } \\
\text { Unclassified }\end{array}$ & UU & 46 & $\begin{array}{l}\text { 19b. TELEPHONE NUMBER (Include area code) } \\
301-394-1523\end{array}$ \\
\hline
\end{tabular}




\section{Contents}

List of Figures $\quad$ iv

$\begin{array}{lr}\text { 1. Introduction } & 1\end{array}$

2. Solution of the Boundary Value Problem $\quad 2$

3. Z-directed Electric and Magnetic Fields 6

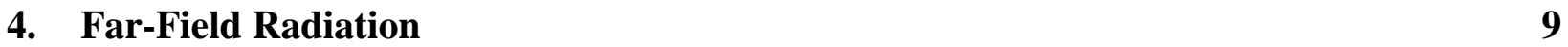

5. Surface Wave Fields $\quad 11$

6. Radiated Power, Directivity, and Efficiency 16

$\begin{array}{lr}\text { 7. Conclusion } & 28\end{array}$

8. References $\quad 29$

$\begin{array}{ll}\text { Appendix A. Boundary Conditions } & 31\end{array}$

$\begin{array}{ll}\text { Appendix B. Branch Cuts } & 35\end{array}$

$\begin{array}{ll}\text { Distribution List } & 40\end{array}$ 


\section{List of Figures}

Fig. 1 The boundary value problem ..............................................................................2

Fig. 2 Branch cuts and behavior of kz1 for this choice in the complex kt plane .....................14

Fig. 3 The closed contour that we use.............................................................................. 14

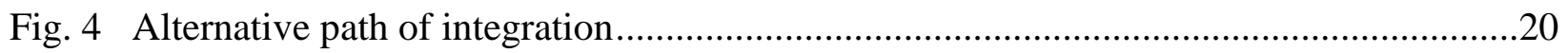

Fig. 5 Equivalent resistances of a horizontal infinitesimal dipole situated on a dielectric substrate; $\varepsilon r=1.01 ; \mu \mathrm{r}=1.01 ; \mathrm{l}=\lambda 0 / 50$

Fig. 6 Directivity of a horizontal dipole situated on a dielectric substrate; $\varepsilon r=1.01$; $\mu \mathrm{r}=$ $1.01 ; 1=\lambda 0 / 50$

Fig. 7 Efficiency of a horizontal infinitesimal dipole situated on a dielectric substrate; $\varepsilon \mathrm{r}=$ $1.01 ; \mu \mathrm{r}=1.01 ; \mathrm{l}=\lambda 0 / 50$

Fig. 8 Equivalent resistances of a horizontal infinitesimal dipole situated on a dielectric substrate; $\varepsilon r=10 ; \mu r=1.01 ; l=\lambda 0 / 50$.

Fig. 9 Directivity of a horizontal infinitesimal dipole situated on a dielectric substrate; $\varepsilon \mathrm{r}=$ $10 ; \mu r=1.01 ; 1=\lambda 0 / 50$

Fig. 10 Efficiency of a horizontal infinitesimal dipole situated on a dielectric substrate; $\varepsilon r=$ $10 ; \mu \mathrm{r}=1.01 ; \mathrm{l}=\lambda 0 / 50$

Fig. 11 Equivalent resistances of a horizontal infinitesimal dipole situated on a dielectric substrate; $\varepsilon r=1.01 ; \mu r=10 ; \mathrm{l}=\lambda 0 / 50$.

Fig. 12 Directivity of a horizontal infinitesimal dipole situated on a dielectric substrate; $\varepsilon r=1.01 ; \mu \mathrm{r}=10 ; \mathrm{l}=\lambda 0 / 50$

Fig. 13 Efficiency of a horizontal infinitesimal dipole situated on a dielectric substrate; $\varepsilon r=$ $1.01 ; \mu \mathrm{r}=10 ; \mathrm{l}=\lambda 0 / 50$ .26

Fig. 14 Equivalent resistances of a horizontal infinitesimal dipole situated on a dielectric substrate; $\varepsilon r=10 ; \mu r=10 ; l=\lambda 0 / 50$

Fig. 15 Directivity of a horizontal infinitesimal dipole situated on a dielectric substrate; $\varepsilon r=10 ; \mu r=10 ; l=\lambda 0 / 50$.

Fig. 16 Efficiency of a horizontal infinitesimal dipole situated on a dielectric substrate; $\varepsilon \mathrm{r}=$ $10 ; \mu \mathrm{r}=10 ; \mathrm{l}=\lambda 0 / 50$. 


\section{Introduction}

This report provides all of the necessary details of the analysis of dipole antennas printed on grounded magnetodielectric substrates. Since the sources considered are essentially impulses, suitable Green's functions could be defined from the formulas contained to facilitate analysis of any radiating sources by superposition. The interest in magnetodielectric substrates stems from the expectation that new materials possessing specially tailored magnetic properties may revolutionize the engineering of thin planar antennas. Since these materials often require complicated constitutive equations to describe their properties rigorously, the solution of intricate boundary value problems has become important to the understanding of the fundamental properties of associated radiating structures.

The boundary value problem considered in this report is simplified substantially by assuming that the magnetodielectric may be modeled as a simple material with scalar constitutive parameters. This approximation is likely not entirely satisfactory; however, it does reduce the problem under consideration to a well-known boundary value problem in applied electromagnetics. Despite the extensive amount of existing literature treating this problem, no completely self-contained treatment of the mathematical boundary value problem itself was found. For this reason, this problem is given a full treatment to demonstrate the method of attack, which may, in future research, be extended to treat the case of magnetic substrates described by a more realistic set of constitutive equations.

Following Arnold Sommerfeld, we pose the boundary value problem in terms of magnetic vector potential and proceed to solve for the unknown coefficients by applying the boundary conditions (the complete derivation of which may be found in Appendix A). In the second section, the electromagnetic field is calculated from the magnetic vector potential. Here, we expend some additional effort to reduce the form of solution to an especially compact and convenient form. These solutions may be compared for accuracy to the solutions reported in the references. Sections 3-6 are devoted to further analysis and manipulation of the solutions to obtain results useful in practice such as surface wave excitation, far-field radiation, directivity, radiation resistance, and efficiency. Finally, it should be noted that a novel approach is used in the treatment of the complex-valued square root functions in the theory of complex integration used to derive the surface waves excited on the magnetodielectric substrate. Appendix B shows how a conventional definition for the square roots can be given, which can be shown to provide all of the necessary (and sufficient) properties to enable a contour to be closed in the lower half complex plane. 


\section{Solution of the Boundary Value Problem}

The boundary value problem is illustrated in Fig. 1 .

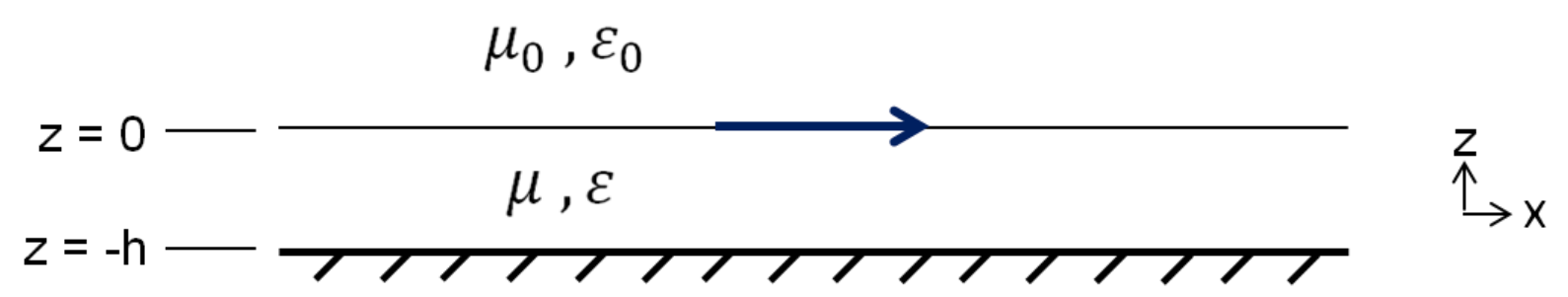

Fig. 1 The boundary value problem

Here we have an $\mathrm{x}$-directed Hertzian dipole situated at an air-dielectric interface. The dielectric layer has a thickness $\mathrm{h}$ and is backed by a perfect electric conductor (PEC) plane located at $\mathrm{z}=-\mathrm{h}$. The current density associated with the Hertzian dipole is

$$
\boldsymbol{J}=\widehat{\boldsymbol{x}} \ell I_{0} \delta(\boldsymbol{r})
$$

The electromagnetic field generated by this current can be represented by 2 components of vector magnetic potential: ${ }^{1}$

$$
\boldsymbol{A}=\widehat{\boldsymbol{x}} A_{x}+\hat{\mathbf{z}} A_{z}
$$

which satisfies the homogeneous Helmholtz equation in both regions, but not at the $\mathrm{z}=0$ interface:

$$
\left(\nabla^{2}+k^{2}\right) \boldsymbol{A}=0
$$

A general solution to Eq. 3 may be obtained for the Cartesian components of $\boldsymbol{A}$ :

$$
\begin{gathered}
A_{u}=\iint_{-\infty}^{\infty} \widetilde{A_{u}} e^{-j k_{x} x} e^{-j k_{y} y} d k_{x} d k_{y} \\
\widetilde{A_{u}}=a e^{-j k_{z} z}+b e^{j k_{z} z} \\
k_{z}=\sqrt{k^{2}-k_{x}{ }^{2}-k_{y}{ }^{2}}
\end{gathered}
$$

The solution to the problem consists of finding the coefficients $(a, b)$ that satisfy all the boundary conditions. The geometry of the current problem suggests that we set

$$
\begin{array}{cc}
\widetilde{A_{x_{1}}}=a e^{-j k_{z_{1}} z} & , \quad z>0 \\
\widetilde{A_{z_{1}}}=b e^{-j k_{z_{1}} z}, & z>0 \\
\widetilde{A_{x_{2}}}=c e^{-j k_{z_{2}} z}+d e^{j k_{z_{2}} z} \quad, \quad & -h<z<0
\end{array}
$$




$$
\widetilde{A_{z_{2}}}=f e^{-j k_{z_{2}} z}+g e^{j k_{z_{2}} z} \quad, \quad-h<z<0 .
$$

In order to determine all of the unknown coefficients in Eq. 5, we require 6 boundary conditions. The derivation of these conditions is somewhat involved. They are listed below (see Appendix A for details).

$$
\begin{gathered}
\left.\widetilde{A_{x_{2}}}\right|_{z=-h}=0 \\
\left.\frac{\partial \widetilde{A_{z_{2}}}}{\partial z}\right|_{z=-h}=0 \\
\left.\widetilde{A_{z_{1}}}\right|_{z=0^{+}}=\left.\frac{1}{\mu_{r}} \widetilde{A_{z_{2}}}\right|_{z=0^{-}} \\
\left.\frac{\partial \widetilde{A_{x_{1}}}}{\partial z}\right|_{z=0^{+}}-\left.\frac{1}{\mu_{r}} \frac{\partial \widetilde{A_{x_{2}}}}{\partial z}\right|_{z=0^{-}}=-\mu_{0} \ell I_{0} \\
\left.\widetilde{A_{x_{1}}}\right|_{z=0^{+}}=\left.\widetilde{A_{x_{2}}}\right|_{z=0^{-}} \\
\left.\frac{\partial \widetilde{A_{z_{1}}}}{\partial z}\right|_{z=0^{+}}-\left.\frac{1}{\mu_{r} \varepsilon_{r}} \frac{\partial \widetilde{A_{z_{2}}}}{\partial z}\right|_{z=0^{-}}=\left.j k_{x} \frac{\mu_{r} \varepsilon_{r}-1}{\mu_{r} \varepsilon_{r}} \widetilde{A_{x_{1}}}\right|_{z=0^{\prime}} .
\end{gathered}
$$

Using the expression for $\widetilde{A_{x_{2}}}$ in Eq. 6, we find

$$
\begin{gathered}
c e^{j k_{z_{2}} h}+d e^{-j k_{z_{2}} h}=0 \\
d=-c e^{2 j k_{z_{2}} h} .
\end{gathered}
$$

Similarly, from Eq. 7,

$$
\begin{gathered}
-j k_{z_{2}} f e^{j k_{z_{2}} h}+j k_{z_{2}} g e^{-j k_{z_{2}} h}=0 \\
g=f e^{2 j k_{z_{2}} h} .
\end{gathered}
$$

Using Eqs. 12 and 13, we can return to Eq. 5 and update our expressions as follows:

$$
\begin{gathered}
\widetilde{A_{x_{1}}}=a e^{-j k_{z_{1}} z} \quad, \quad z>0 \\
\widetilde{A_{z_{1}}}=b e^{-j k_{z_{1}} z} \quad, \quad z>0 \\
\widetilde{A_{x_{2}}}=c\left(e^{-j k_{z_{2}} z}-e^{2 j k_{z_{2}} h} e^{j k_{z_{2}} z}\right) \quad, \quad-h<z<0 \\
\widetilde{A_{z_{2}}}=f\left(e^{-j k_{z_{2}} z}+e^{2 j k_{z_{2}} h} e^{j k_{z_{2}} z}\right) \quad, \quad-h<z<0 .
\end{gathered}
$$

Next, we plug the updated expressions Eq. 14 into the boundary condition Eq. 9:

$$
-j k_{z_{1}} a-\frac{1}{\mu_{r}} c\left(-j k_{z_{2}}-j k_{z_{2}} e^{2 j k_{z_{2}} h}\right)=-\mu_{0} \ell I_{0} .
$$

Similarly, from Eq. 10,

$$
a=c\left(1-e^{2 j k_{z_{2}} h}\right) .
$$

Substituting Eq. 16 into Eq. 15, 


$$
\begin{gathered}
-j k_{z_{1}} c\left(1-e^{2 j k_{z_{2}} h}\right)+\frac{1}{\mu_{r}} j k_{z_{2}} c\left(1+e^{2 j k_{z_{2}} h}\right)=-\mu_{0} \ell I_{0} \\
-j k_{z_{1}} c e^{j k_{z_{2}} h}\left(e^{-j k_{z_{2}} h}-e^{j k_{z_{2}} h}\right)+\frac{1}{\mu_{r}} j k_{z_{2}} c e^{j k_{z_{2}} h}\left(e^{-j k_{z_{2}} h}+e^{j k_{z_{2}} h}\right)=-\mu_{0} \ell I_{0} \\
-2 k_{z_{1}} c e^{j k_{z_{2}} h} \sin \left(k_{z_{2}} h\right)+\frac{2}{\mu_{r}} j k_{z_{2}} c e^{j k_{z_{2}} h} \cos \left(k_{z_{2}} h\right)=-\mu_{0} \ell I_{0} \\
2 j c \sin \left(k_{z_{2}} h\right) e^{j k_{z_{2}} h}\left[\mu_{r} j k_{z_{1}}+k_{z_{2}} \cot \left(k_{z_{2}} h\right)\right]=-\mu_{0} \mu_{r} \ell I_{0}
\end{gathered}
$$

We denote the bracketed expression in Eq. 17 as follows: ${ }^{2}$

$$
D_{T E}=\mu_{r} j k_{z_{1}}+k_{z_{2}} \cot \left(k_{z_{2}} h\right)
$$

Then,

$$
c=\frac{-\mu_{0} \mu_{r} \ell I_{0}}{2 j D_{T E} \sin \left(k_{z_{2}} h\right) e^{j k_{z_{2}} h}}
$$

Substitution of Eq. 19 into Eq. 16 gives

$$
\begin{gathered}
a=\frac{-\mu_{0} \mu_{r} \ell I_{0}}{2 j D_{T E} \sin \left(k_{z_{2}} h\right) e^{j k_{z_{2}} h}}\left(1-e^{2 j k_{z_{2}} h}\right) \\
a=\frac{-\mu_{0} \mu_{r} \ell I_{0}}{2 j D_{T E} \sin \left(k_{z_{2}} h\right) e^{j k_{z_{2}} h} e^{j k_{z_{2}} h}\left(e^{-j k_{z_{2}} h}-e^{j k_{z_{2}} h}\right)} \\
a=\frac{\mu_{0} \mu_{r} \ell I_{0}}{D_{T E}}
\end{gathered}
$$

Next, we apply boundary condition Eq. 8, obtaining

$$
b=\frac{1}{\mu_{r}} f\left(1+e^{2 j k_{z_{2}} h}\right)
$$

and, from boundary condition Eq. 11,

$$
-j k_{z_{1}} b-\frac{1}{\mu_{r} \varepsilon_{r}} f\left(-j k_{z_{2}}+j k_{z_{2}} e^{2 j k_{z_{2}} h}\right)=j k_{x} \frac{\mu_{r} \varepsilon_{r}-1}{\mu_{r} \varepsilon_{r}} a
$$

Substitution of Eqs. 20 and 21 into Eq. 22 gives

$$
\begin{gathered}
-j k_{z_{1}} \frac{1}{\mu_{r}} f\left(1+e^{2 j k_{z_{2}} h}\right)-\frac{1}{\mu_{r} \varepsilon_{r}} f\left(-j k_{z_{2}}+j k_{z_{2}} e^{2 j k_{z_{2}} h}\right)=j k_{x} \frac{\mu_{r} \varepsilon_{r}-1}{\mu_{r} \varepsilon_{r}} \frac{\mu_{0} \mu_{r} \ell I_{0}}{D_{T E}} \\
-j k_{z_{1}} \frac{1}{\mu_{r}} f e^{j k_{z_{2}} h}\left(e^{-j k_{z_{2}} h}+e^{j k_{z_{2}} h}\right)+j k_{z_{2}} \frac{1}{\mu_{r} \varepsilon_{r}} f e^{j k_{z_{2}} h}\left(e^{-j k_{z_{2}} h}-e^{j k_{z_{2}} h}\right) \\
=j k_{x} \frac{\mu_{r} \varepsilon_{r}-1}{\mu_{r} \varepsilon_{r}} \frac{\mu_{0} \mu_{r} \ell I_{0}}{D_{T E}} \\
-2 j k_{z_{1}} \frac{1}{\mu_{r}} f e^{j k_{z_{2}} h} \cos \left(k_{z_{2}} h\right)+2 k_{z_{2}} \frac{1}{\mu_{r} \varepsilon_{r}} f e^{j k_{z_{2}} h} \sin \left(k_{z_{2}} h\right)=j k_{x} \frac{\mu_{r} \varepsilon_{r}-1}{\mu_{r} \varepsilon_{r}} \frac{\mu_{0} \mu_{r} \ell I_{0}}{D_{T E}} \\
\frac{-2 f \cos \left(k_{z_{2}} h\right) e^{j k_{z_{2}} h}}{\mu_{r} \varepsilon_{r}}\left[\varepsilon_{r} j k_{z_{1}}-k_{z_{2}} \tan \left(k_{z_{2}} h\right)\right]=j k_{x} \frac{\mu_{r} \varepsilon_{r}-1}{\mu_{r} \varepsilon_{r}} \frac{\mu_{0} \mu_{r} \ell I_{0}}{D_{T E}}
\end{gathered}
$$


We denote the bracketed expression in Eq. 23 as follows: ${ }^{2}$

$$
D_{T M}=\varepsilon_{r} j k_{z_{1}}-k_{z_{2}} \tan \left(k_{z_{2}} h\right)
$$

Then,

$$
f=\frac{-\mu_{0} \mu_{r} \ell I_{0} j k_{x}\left(\mu_{r} \varepsilon_{r}-1\right)}{2 D_{T E} D_{T M} \cos \left(k_{z_{2}} h\right) e^{j k_{z_{2}} h}}
$$

Substitution of Eq. 25 into Eq. 21 gives

$$
\begin{gathered}
b=\frac{1}{\mu_{r}} \frac{-\mu_{0} \mu_{r} \ell I_{0} j k_{x}\left(\mu_{r} \varepsilon_{r}-1\right)}{2 D_{T E} D_{T M} \cos \left(k_{z_{2}} h\right) e^{j k_{z_{2}} h}}\left(1+e^{2 j k_{z_{2}} h}\right) \\
b=\frac{-\mu_{0} \ell I_{0} j k_{x}\left(\mu_{r} \varepsilon_{r}-1\right)}{2 D_{T E} D_{T M} \cos \left(k_{z_{2}} h\right) e^{j k_{z_{2}} h} e^{j k_{z_{2}} h}\left(e^{-j k_{z_{2}} h}+e^{j k_{z_{2}} h}\right)} \\
b=\frac{-\mu_{0} \ell I_{0} j k_{x}\left(\mu_{r} \varepsilon_{r}-1\right)}{D_{T E} D_{T M}}
\end{gathered}
$$

This completes the solution for the unknown coefficients. We can now return to Eq. 14 and write

$$
\begin{gathered}
\widetilde{A_{x_{2}}}=\frac{-\mu_{0} \mu_{r} \ell I_{0}}{2 j D_{T E} \sin \left(k_{z_{2}} h\right) e^{j k_{z_{2}} h}}\left(e^{-j k_{z_{2}} z}-e^{2 j k_{z_{2}} h} e^{j k_{z_{2}} z}\right) \\
\widetilde{A_{x_{2}}}=\frac{-\mu_{0} \mu_{r} \ell I_{0}}{2 j D_{T E} \sin \left(k_{z_{2}} h\right) e^{j k_{z_{2}} h} e^{j k_{z_{2}} h}\left(e^{-j k_{z_{2}}(z+h)}-e^{j k_{z_{2}}(z+h)}\right)} \\
\widetilde{A_{x_{2}}}=\frac{\mu_{0} \mu_{r} \ell I_{0} \sin \left[k_{z_{2}}(z+h)\right]}{D_{T E} \sin \left(k_{z_{2}} h\right)}
\end{gathered}
$$

Similarly,

$$
\begin{gathered}
\widetilde{A_{z_{2}}}=\frac{-\mu_{0} \mu_{r} \ell I_{0} j k_{x}\left(\mu_{r} \varepsilon_{r}-1\right)}{2 D_{T E} D_{T M} \cos \left(k_{z_{2}} h\right) e^{j k_{z_{2}} h}}\left(e^{-j k_{z_{2}} z}+e^{2 j k_{z_{2}} h} e^{j k_{z_{2}} z}\right) \\
\widetilde{A_{z_{2}}}=\frac{-\mu_{0} \mu_{r} \ell I_{0} j k_{x}\left(\mu_{r} \varepsilon_{r}-1\right) \cos \left[k_{z_{2}}(z+h)\right]}{D_{T E} D_{T M} \cos \left(k_{z_{2}} h\right)}
\end{gathered}
$$


Assembling all of our results, the complete solution to the boundary value problem is

$$
\begin{gathered}
\widetilde{A_{x_{1}}}=\frac{\mu_{0} \mu_{r} \ell I_{0}}{D_{T E}} e^{-j k_{z_{1}} z}, \quad z>0 \\
\widetilde{A_{z_{1}}}=\frac{-\mu_{0} \ell I_{0} j k_{x}\left(\mu_{r} \varepsilon_{r}-1\right)}{D_{T E} D_{T M}} e^{-j k_{z_{1}} z}, \quad z>0 \\
\widetilde{A_{x_{2}}}=\frac{\mu_{0} \mu_{r} \ell I_{0}}{D_{T E} \sin \left(k_{z_{2}} h\right)} \sin \left[k_{z_{2}}(z+h)\right], \quad-h<z<0 \\
\widetilde{A_{z_{2}}}=\frac{-\mu_{0} \mu_{r} \ell I_{0} j k_{x}\left(\mu_{r} \varepsilon_{r}-1\right)}{D_{T E} D_{T M} \cos \left(k_{z_{2}} h\right)} \cos \left[k_{z_{2}}(z+h)\right], \quad-h<z<0 \\
D_{T E}=\mu_{r} j k_{z_{1}}+k_{z_{2}} \cot \left(k_{z_{2}} h\right) \\
D_{T M}=\varepsilon_{r} j k_{z_{1}}-k_{z_{2}} \tan \left(k_{z_{2}} h\right) \\
k_{z_{1}}=\sqrt{k_{0}{ }^{2}-k_{x}{ }^{2}-k_{y}{ }^{2}} \\
k_{z_{2}}=\sqrt{\mu_{r} \varepsilon_{r} k_{0}{ }^{2}-k_{x}{ }^{2}-k_{y}{ }^{2}}
\end{gathered}
$$

Note that a 2-dimensional inverse Fourier transform is required to recover the spatial dependence of the fields. It is also worth mentioning here that $D_{T E}$ and $D_{T M}$ represent the dispersion equations for supported surface wave modes that are transverse electric (TE) and transverse magnetic (TM), respectively, to the $\hat{\mathbf{z}}$ axis. As such, our next step is to derive the z-components of the electric and magnetic fields. Most quantities of interest can be calculated directly from these fields.

\section{Z-directed Electric and Magnetic Fields}

The z-components of the electric and magnetic fields are related to the magnetic vector potential as follows:

$$
\begin{gathered}
\widetilde{H_{z}}=\frac{j k_{y}}{\mu} \widetilde{A_{x}} \\
\widetilde{E_{z}}=\frac{1}{j \omega \mu \varepsilon}\left[-j k_{x} \frac{\widetilde{\partial A_{x}}}{\partial z}+\left(k_{x}{ }^{2}+k_{y}{ }^{2}\right) \widetilde{A_{z}}\right]
\end{gathered}
$$

The equation for the z-directed magnetic field is rather simple. Using our results from Eq. 29, we obtain 


$$
\begin{gathered}
\widetilde{H_{z_{1}}}=\frac{\ell I_{0} \mu_{r} j k_{y}}{D_{T E}} e^{-j k_{z_{1}} z}, \quad z>0 \\
\widetilde{H_{z_{2}}}=\frac{\ell I_{0} j k_{y}}{D_{T E} \sin \left(k_{z_{2}} h\right)} \sin \left[k_{z_{2}}(z+h)\right],-h<z<0
\end{gathered}
$$

Next, focusing on the z-directed electric field for $z>0$, we first write

$$
-j k_{x} \frac{\widetilde{\partial A_{x_{1}}}}{\partial z}=\frac{-\mu_{0} \mu_{r} \ell I_{0} k_{x} k_{z_{1}}}{D_{T E}} e^{-j k_{z_{1}} z}
$$

Substitution of Eq. 32 and the expression for $\widetilde{A_{z_{1}}}$ from Eq. 29 into Eq. 30 yields

$$
\widetilde{E_{z_{1}}}=\frac{\ell I_{0} \eta_{0} j k_{x}}{k_{0} D_{T E} D_{T M}} e^{-j k_{z_{1}} z}\left[j\left(\mu_{r} \varepsilon_{r}-1\right)\left(k_{\chi}{ }^{2}+k_{y}{ }^{2}\right)+\mu_{r} k_{z_{1}} D_{T M}\right]
$$

Although it is difficult to tell, the form of Eq. 33 can actually be considerably simplified. In a (non-obvious) step,

$$
\begin{gathered}
\left(\mu_{r} \varepsilon_{r}-1\right)\left(k_{x}{ }^{2}+{k_{y}}^{2}\right)=\mu_{r} \varepsilon_{r}\left({k_{x}}^{2}+{k_{y}}^{2}\right)-\left({k_{x}}^{2}+{k_{y}}^{2}\right) \\
\left(\mu_{r} \varepsilon_{r}-1\right)\left(k_{x}{ }^{2}+k_{y}{ }^{2}\right)=\mu_{r} \varepsilon_{r}\left({k_{0}}^{2}-{k_{z_{1}}}^{2}\right)-\left(\mu_{r} \varepsilon_{r}{k_{0}}^{2}-{k_{z_{2}}}^{2}\right) \\
\left(\mu_{r} \varepsilon_{r}-1\right)\left(k_{x}{ }^{2}+{k_{y}}^{2}\right)={k_{z_{2}}}^{2}-\mu_{r} \varepsilon_{r}{k_{z_{1}}}^{2}
\end{gathered}
$$

We substitute Eq. 34 into Eq. 33:

$$
\begin{gathered}
\widetilde{E_{z_{1}}}=\frac{\ell I_{0} \eta_{0} j k_{x}}{k_{0} D_{T E} D_{T M}} e^{-j k_{z_{1}} z}\left[j\left(k_{z_{2}}{ }^{2}-\mu_{r} \varepsilon_{r}{k_{z_{1}}}^{2}\right)+\mu_{r} k_{z_{1}} D_{T M}\right] \\
\widetilde{E_{z_{1}}}=\frac{\ell I_{0} \eta_{0} j k_{x}}{k_{0} D_{T E} D_{T M}} e^{-j k_{z_{1}} z}\left[j{z_{z_{2}}}^{2}+\mu_{r} k_{z_{1}}\left(D_{T M}-\varepsilon_{r} j k_{z_{1}}\right)\right] \\
\widetilde{E_{z_{1}}}=\frac{\ell I_{0} \eta_{0} j k_{x}}{k_{0} D_{T E} D_{T M}} e^{-j k_{z_{1}} z}\left[j k_{z_{2}}{ }^{2}+\mu_{r} k_{z_{1}}\left(\varepsilon_{r} j k_{z_{1}}-k_{z_{2}} \tan \left(k_{z_{2}} h\right)-\varepsilon_{r} j k_{z_{1}}\right)\right] \\
\widetilde{E_{z_{1}}}=\frac{\ell I_{0} \eta_{0} j k_{x}}{k_{0} D_{T E} D_{T M}} e^{-j k_{z_{1}} z}\left[j k_{z_{2}}{ }^{2}-\mu_{r} k_{z_{1}} k_{z_{2}} \tan \left(k_{z_{2}} h\right)\right] \\
\widetilde{E_{z_{1}}}=-\frac{\ell I_{0} \eta_{0} k_{x} k_{z_{2}}}{k_{0} D_{T E} D_{T M}} \tan \left(k_{z_{2}} h\right) e^{-j k_{z_{1}} z}\left[k_{z_{2}} \cot \left(k_{z_{2}} h\right)+\mu_{r} j k_{z_{1}}\right] \\
\widetilde{E_{z_{1}}}=-\frac{\ell I_{0} \eta_{0} k_{x} k_{z_{2}}}{k_{0} D_{T E} D_{T M}} \tan \left(k_{z_{2}} h\right) e^{-j k_{z_{1}} z}\left[D_{T E}\right] \\
\widetilde{E_{z_{1}}}=-\frac{\ell I_{0} \eta_{0} k_{x} k_{z_{2}}}{k_{0} D_{T M}} \tan \left(k_{z_{2}} h\right) e^{-j k_{z_{1}} z}, \quad, z>0
\end{gathered}
$$


Lastly, we must find the z-directed electric field for $-h<z<0$. As before, we first write

$$
-j k_{x} \frac{\widetilde{\partial A_{x_{2}}}}{\partial z}=\frac{-\mu_{0} \mu_{r} \ell I_{0} j k_{x} k_{z_{2}}}{D_{T E} \sin \left(k_{z_{2}} h\right)} \cos \left[k_{z_{2}}(z+h)\right]
$$

Substitution of Eq. 36 and the expression for $\widetilde{A_{z_{2}}}$ from Eq. 29 into Eq. 30 yields

$$
\widetilde{E_{z_{2}}}=\frac{-\mu_{0} \mu_{r} \ell I_{0} j k_{x}}{j \omega \mu_{0} \mu_{r} \varepsilon_{0} \varepsilon_{r} D_{T E}} \cos \left[k_{z_{2}}(z+h)\right]\left[\frac{\left(\mu_{r} \varepsilon_{r}-1\right)\left(k_{x}{ }^{2}+k_{y}{ }^{2}\right)}{D_{T M} \cos \left(k_{z_{2}} h\right)}+\frac{k_{z_{2}}}{\sin \left(k_{z_{2}} h\right)}\right] .
$$

As it was for $\widetilde{E_{z_{1}}}$, we can also simplify Eq. 37 considerably:

$$
\begin{aligned}
& \widetilde{E_{z_{2}}}=\frac{-\ell I_{0} k_{x}}{\omega \varepsilon_{0} \varepsilon_{r} D_{T E} D_{T M}} \frac{\cos \left[k_{z_{2}}(z+h)\right]}{\cos \left(k_{z_{2}} h\right)}\left[\left(\mu_{r} \varepsilon_{r}-1\right)\left(k_{x}{ }^{2}+k_{y}{ }^{2}\right)+D_{T M} k_{z_{2}} \cot \left(k_{z_{2}} h\right)\right] \\
& \widetilde{E_{z_{2}}}=\frac{-\ell I_{0} k_{x}}{\omega \varepsilon_{0} \varepsilon_{r} D_{T E} D_{T M}} \frac{\cos \left[k_{z_{2}}(z+h)\right]}{\cos \left(k_{z_{2}} h\right)}\left[\left(k_{z_{2}}{ }^{2}-\mu_{r} \varepsilon_{r} k_{z_{1}}{ }^{2}\right)\right. \\
& \left.+\left(\varepsilon_{r} j k_{z_{1}}-k_{z_{2}} \tan \left(k_{z_{2}} h\right)\right) k_{z_{2}} \cot \left(k_{z_{2}} h\right)\right] \\
& \widetilde{E_{z_{2}}}=\frac{-\ell I_{0} k_{x}}{\omega \varepsilon_{0} \varepsilon_{r} D_{T E} D_{T M}} \frac{\cos \left[k_{z_{2}}(z+h)\right]}{\cos \left(k_{z_{2}} h\right)}\left[k_{z_{2}}{ }^{2}-\mu_{r} \varepsilon_{r} k_{z_{1}}{ }^{2}+\varepsilon_{r} j k_{z_{1}} k_{z_{2}} \cot \left(k_{z_{2}} h\right)-k_{z_{2}}{ }^{2}\right] \\
& \widetilde{E_{z_{2}}}=\frac{-\ell I_{0} k_{x}}{\omega \varepsilon_{0} \varepsilon_{r} D_{T E} D_{T M}} \frac{\cos \left[k_{z_{2}}(z+h)\right]}{\cos \left(k_{z_{2}} h\right)}\left[\mu_{r} \varepsilon_{r} k_{z_{1}}{ }^{2}+\varepsilon_{r} j k_{z_{1}} k_{z_{2}} \cot \left(k_{z_{2}} h\right)\right] \\
& \widetilde{E_{z_{2}}}=\frac{-\varepsilon_{r} \ell I_{0} k_{x} k_{z_{1}}}{\omega \varepsilon_{0} \varepsilon_{r} D_{T E} D_{T M}} \frac{\cos \left[k_{z_{2}}(z+h)\right]}{\cos \left(k_{z_{2}} h\right)}\left[\mu_{r} k_{z_{1}}+j k_{z_{2}} \cot \left(k_{z_{2}} h\right)\right] \\
& \widetilde{E_{z_{2}}}=\frac{-\ell I_{0} j k_{x} k_{z_{1}}}{\omega \varepsilon_{0} D_{T E} D_{T M}} \frac{\cos \left[k_{z_{2}}(z+h)\right]}{\cos \left(k_{z_{2}} h\right)}\left[\mu_{r} j k_{z_{1}}+k_{z_{2}} \cot \left(k_{z_{2}} h\right)\right] \\
& \widetilde{E_{z_{2}}}=\frac{-\ell I_{0} j k_{x} k_{z_{1}}}{\omega \varepsilon_{0} D_{T E} D_{T M}} \frac{\cos \left[k_{z_{2}}(z+h)\right]}{\cos \left(k_{z_{2}} h\right)}\left[D_{T E}\right] \\
& \widetilde{E_{z_{2}}}=-\frac{\ell I_{0} \eta_{0} j k_{x} k_{z_{1}}}{k_{0} D_{T M}} \frac{\cos \left[k_{z_{2}}(z+h)\right]}{\cos \left(k_{z_{2}} h\right)} \quad, \quad-h<z<0
\end{aligned}
$$


Assembling these results, we get the following:

$$
\begin{gathered}
\widetilde{H_{z_{1}}}=\frac{\ell I_{0} \mu_{r} j k_{y}}{D_{T E}} e^{-j k_{z_{1}} z}, \quad z>0 \\
\widetilde{H_{z_{2}}}=\frac{\ell I_{0} j k_{y}}{D_{T E} \sin \left(k_{z_{2}} h\right)} \sin \left[k_{z_{2}}(z+h)\right], \quad-h<z<0 \\
\widetilde{E_{z_{1}}}=-\frac{\ell I_{0} \eta_{0} k_{x} k_{z_{2}}}{k_{0} D_{T M}} \tan \left(k_{z_{2}} h\right) e^{-j k_{z_{1}} z}, \quad z>0 \\
\widetilde{E_{z_{2}}}=-\frac{\ell I_{0} \eta_{0} j k_{x} k_{z_{1}}}{k_{0} D_{T M}} \frac{\cos \left[k_{z_{2}}(z+h)\right]}{\cos \left(k_{z_{2}} h\right)}, \quad-h<z<0
\end{gathered}
$$

As mentioned previously, in order to determine the actual spatial dependence of the electric and magnetic fields, we must perform the inverse 2-dimensional Fourier transform of the expressions in Eq. 39. Unfortunately, it appears to be impossible to integrate Eq. 39 in closed form. However, far from the Hertzian dipole, we can employ the stationary phase approximation to the integrands to obtain the far-fields. Additionally, it is also possible to extract TE and TM surface wave fields by interpreting the integral as a contour integral in the complex $k_{x}$ and $k_{y}$ domains.

\section{Far-Field Radiation}

From Eq. 39, we could, for instance, write the solution for the z-directed electric field above the substrate as

$$
\begin{gathered}
E_{z_{1}}=\frac{1}{(2 \pi)^{2}} \iint_{-\infty}^{\infty} \widetilde{E_{z_{1}}} e^{-j k_{x} x} e^{-j k_{y} y} d k_{x} d k_{y} \\
E_{z_{1}} \\
=-\frac{1}{(2 \pi)^{2}} \iint_{-\infty}^{\infty} \frac{\ell I_{0} \eta_{0} k_{x} k_{z_{2}}}{k_{0} D_{T M}} \tan \left(k_{z_{2}} h\right) e^{-j k_{z_{1}} z} e^{-j k_{x} x} e^{-j k_{y} y} d k_{x} d k_{y}
\end{gathered}
$$

The integral Eq. 40 is of a general form for which the stationary phase approximation is well known: ${ }^{3}$

$$
\left.E_{z_{1}} \approx-\frac{\ell I_{0} \eta_{0} j k_{x_{s}} k_{z_{2_{s}}} \cos \theta}{k_{0} D_{T M_{s}}} \tan \left(k_{z_{2_{s}}} h\right) \frac{e^{-j k_{0} r}}{2 \pi r}, \text { (far field }\right) \text {, }
$$

where 


$$
\begin{gathered}
k_{x_{s}}=k_{0} \sin \theta \cos \phi \\
k_{y_{s}}=k_{0} \sin \theta \sin \phi \\
k_{z_{1_{s}}}=k_{0} \cos \theta \\
k_{z_{2_{s}}}=k_{0} \sqrt{\mu_{r} \varepsilon_{r}-(\sin \theta)^{2}} \\
D_{T E_{s}}=\mu_{r} j k_{z_{1_{s}}}+k_{z_{2_{s}}} \cot \left(k_{z_{2_{s}}} h\right) \\
D_{T M_{s}}=\varepsilon_{r} j k_{z_{1_{s}}}-k_{z_{z_{s}}} \tan \left(k_{z_{2_{s}}} h\right)
\end{gathered}
$$

Similarly,

$$
\begin{gathered}
H_{z_{1}}=\frac{1}{(2 \pi)^{2}} \iint_{-\infty}^{\infty} \widetilde{H_{z_{1}}} e^{-j k_{x} x} e^{-j k_{y} y} d k_{x} d k_{y} \\
H_{z_{1}}=\frac{1}{(2 \pi)^{2}} \iint_{-\infty}^{\infty} \frac{\ell I_{0} \mu_{r} j k_{y}}{D_{T E}} e^{-j k_{z_{1}} z} e^{-j k_{x} x} e^{-j k_{y} y} d k_{x} d k_{y}
\end{gathered}
$$

and

$$
H_{z_{1}} \approx-\frac{\ell I_{0} \mu_{r} k_{y_{s}} \cos \theta}{D_{T E_{s}}} \frac{e^{-j k_{0} r}}{2 \pi r},(\text { far field })
$$

Additionally, under the stationary phase approximation,

$$
\begin{gathered}
E_{r}=0 \\
E_{\theta}=-\frac{E_{z}}{\sin \theta} \\
E_{\phi}=\frac{\eta_{0} H_{z}}{\sin \theta}
\end{gathered}
$$

Therefore, a good approximation to the radiated field in the far-field in the upper hemisphere is

$$
\begin{gathered}
E_{r}=0 \\
E_{\theta}=\frac{\ell I_{0} \eta_{0} j k_{x_{s}} k_{z_{2_{s}}} \cot \theta}{k_{0} D_{T M_{s}}} \tan \left(k_{z_{2_{s}}} h\right) \frac{e^{-j k_{0} r}}{2 \pi r} \\
E_{\phi}=-\frac{\ell I_{0} \eta_{0} \mu_{r} k_{y_{s}} \cot \theta}{D_{T E_{S}}} \frac{e^{-j k_{0} r}}{2 \pi r}
\end{gathered}
$$




\section{Surface Wave Fields}

We now turn toward the derivation of the surface wave fields generated by the Hertzian dipole. In order to do this, we must interpret our integrals as contour integrals in the complex $k_{x}$ and $k_{y}$ domains. For convenience, the integrals are repeated below:

$$
\begin{gathered}
H_{z_{1}}=\frac{1}{(2 \pi)^{2}} \iint_{-\infty}^{\infty} \frac{\ell I_{0} \mu_{r} j k_{y}}{D_{T E}} e^{-j k_{z_{1}} z} e^{-j k_{x} x} e^{-j k_{y} y} d k_{x} d k_{y} \\
H_{z_{2}}=\frac{1}{(2 \pi)^{2}} \iint_{-\infty}^{\infty} \frac{\ell I_{0} j k_{y}}{D_{T E} \sin \left(k_{z_{2}} h\right)} \sin \left[k_{z_{2}}(z+h)\right] e^{-j k_{x} x} e^{-j k_{y} y} d k_{x} d k_{y} \\
E_{z_{1}}=-\frac{1}{(2 \pi)^{2}} \int_{-\infty}^{\infty} \frac{\ell I_{0} \eta_{0} k_{x} k_{z_{2}}}{k_{0} D_{T M}} \tan \left(k_{z_{2}} h\right) e^{-j k_{z_{1}} z} e^{-j k_{x} x} e^{-j k_{y} y} d k_{x} d k_{y} \\
E_{z_{2}}=-\frac{1}{(2 \pi)^{2}} \iint_{-\infty}^{\infty} \frac{\ell I_{0} \eta_{0} j k_{x} k_{z_{1}}}{k_{0} D_{T M}} \frac{\cos \left[k_{z_{2}}(z+h)\right]}{\cos \left(k_{z_{2}} h\right)} e^{-j k_{x} x} e^{-j k_{y} y} d k_{x} d k_{y}
\end{gathered}
$$

We introduce the change of variables,

$$
\begin{gathered}
k_{x}=k_{t} \cos \alpha \\
k_{y}=k_{t} \sin \alpha \\
x=\rho \cos \phi \\
y=\rho \sin \phi
\end{gathered}
$$

Noting that

$$
k_{x} x+k_{y} y=k_{t} \rho(\cos \alpha \cos \phi+\sin \alpha \sin \phi)=k_{t} \rho \cos (\alpha-\phi)
$$


the integrals in Eq. 47 become

$$
\begin{gathered}
H_{z_{1}}=\frac{1}{(2 \pi)^{2}} \int_{0}^{2 \pi} \int_{0}^{\infty} \frac{\ell I_{0} \mu_{r} j k_{t} \sin \alpha}{D_{T E}} e^{-j k_{z_{1}} z} e^{-j k_{t} \rho \cos (\alpha-\phi)} k_{t} d k_{t} d \alpha \\
H_{z_{2}}=\frac{1}{(2 \pi)^{2}} \int_{0}^{2 \pi} \int_{0}^{\infty} \frac{\ell I_{0} j k_{t} \sin \alpha}{D_{T E} \sin \left(k_{z_{2}} h\right)} \sin \left[k_{z_{2}}(z+h)\right] e^{-j k_{t} \rho \cos (\alpha-\phi)} k_{t} d k_{t} d \alpha \\
E_{z_{1}}=-\frac{1}{(2 \pi)^{2}} \int_{0}^{2 \pi} \int_{0}^{\infty} \frac{\ell I_{0} \eta_{0} k_{t} \cos \alpha k_{z_{2}}}{k_{0} D_{T M}} \tan \left(k_{z_{2}} h\right) e^{-j k_{z_{1}} z} e^{-j k_{t} \rho \cos (\alpha-\phi)} k_{t} d k_{t} d \alpha \\
E_{z_{2}}=-\frac{1}{(2 \pi)^{2}} \int_{0}^{2 \pi} \int_{0}^{\infty} \frac{\ell I_{0} \eta_{0} j k_{t} \cos \alpha k_{z_{1}}}{k_{0} D_{T M}} \frac{\cos \left[k_{z_{2}}(z+h)\right]}{\cos \left(k_{z_{2}} h\right)} e^{-j k_{t} \rho \cos (\alpha-\phi)} k_{t} d k_{t} d \alpha
\end{gathered}
$$

With the aid of the following 2 mathematical identities,

$$
\begin{aligned}
& -2 \pi j J_{1}\left(k_{t} \rho\right) \cos \phi=\int_{0}^{2 \pi} \cos \alpha e^{-j k_{t} \rho \cos (\alpha-\phi)} d \alpha \\
& -2 \pi j J_{1}\left(k_{t} \rho\right) \sin \phi=\int_{0}^{2 \pi} \sin \alpha e^{-j k_{t} \rho \cos (\alpha-\phi)} d \alpha
\end{aligned}
$$

the integral expressions in Eq. 50 reduce to

$$
\begin{gathered}
H_{z_{1}}=\frac{\ell I_{0} \mu_{r} \sin \phi}{2 \pi} \int_{0}^{\infty} \frac{k_{t}}{D_{T E}} e^{-j k_{z_{1}} z_{1}} J_{1}\left(k_{t} \rho\right) k_{t} d k_{t} \\
H_{z_{2}}=\frac{\ell I_{0} \sin \phi}{2 \pi} \int_{0}^{\infty} \frac{k_{t}}{D_{T E}} \frac{\sin \left[k_{z_{2}}(z+h)\right]}{\sin \left(k_{z_{2}} h\right)} J_{1}\left(k_{t} \rho\right) k_{t} d k_{t} \\
E_{z_{1}}=\frac{j \ell I_{0} \eta_{0} \cos \phi}{k_{0} 2 \pi} \int_{0}^{\infty} \frac{k_{t} k_{z_{2}}}{D_{T M}} \tan \left(k_{z_{2}} h\right) e^{-j k_{z_{1}} z} J_{1}\left(k_{t} \rho\right) k_{t} d k_{t} \\
E_{z_{2}}=-\frac{\ell I_{0} \eta_{0} \cos \phi}{k_{0} 2 \pi} \int_{0}^{\infty} \frac{k_{t} k_{z_{1}}}{D_{T M}} \frac{\cos \left[k_{z_{2}}(z+h)\right]}{\cos \left(k_{z_{2}} h\right)} J_{1}\left(k_{t} \rho\right) k_{t} d k_{t}
\end{gathered}
$$


Next, for $f\left(k_{t}\right)$, an odd function of $k_{t}^{2}$

$$
\int_{0}^{\infty} f\left(k_{t}\right) J_{1}\left(k_{t} \rho\right) k_{t} d k_{t}=\frac{1}{2} \int_{-\infty}^{\infty} f\left(k_{t}\right) H_{1}^{(2)}\left(k_{t} \rho\right) k_{t} d k_{t}
$$

Using the large argument approximation for the Hankel function ${ }^{3}$

$$
H_{1}^{(2)}\left(k_{t} \rho\right) \approx j \sqrt{\frac{2}{\pi k_{t} \rho}} e^{j \frac{\pi}{4}} e^{-j k_{t} \rho}
$$

we arrive at the approximate form of the integral expressions for the $\mathrm{z}$-components of the electric and magnetic field for large $\rho$ :

$$
\begin{gathered}
H_{z_{1}}=\frac{j \ell I_{0} \mu_{r} \sin \phi}{4 \pi} e^{j \frac{\pi}{4}} \int_{-\infty}^{\infty} \frac{1}{D_{T E}} \sqrt{\frac{2 k_{t}}{\pi \rho}} e^{-j k_{z_{1}} z} e^{-j k_{t} \rho} k_{t} d k_{t} \\
H_{z_{2}}=\frac{j \ell I_{0} \sin \phi}{4 \pi} e^{j \frac{\pi}{4}} \int_{-\infty}^{\infty} \frac{1}{D_{T E}} \sqrt{\frac{2 k_{t}}{\pi \rho}} \frac{\sin \left[k_{z_{2}}(z+h)\right]}{\sin \left(k_{z_{2}} h\right)} e^{-j k_{t} \rho} k_{t} d k_{t} \\
E_{z_{1}}=-\frac{\ell I_{0} \eta_{0} \cos \phi}{k_{0} 4 \pi} e^{j \frac{\pi}{4}} \int_{-\infty}^{\infty} \frac{k_{z_{2}}}{D_{T M}} \sqrt{\frac{2 k_{t}}{\pi \rho}} \tan \left(k_{z_{2}} h\right) e^{-j k_{z_{1}} z} e^{-j k_{t} \rho} k_{t} d k_{t} \\
E_{z_{2}}=-\frac{j \ell I_{0} \eta_{0} \cos \phi}{k_{0} 4 \pi} e^{j \frac{\pi}{4}} \int_{-\infty}^{\infty} \frac{k_{z_{1}}}{D_{T M}} \sqrt{\frac{2 k_{t}}{\pi \rho}} \frac{\cos \left[k_{z_{2}}(z+h)\right]}{\cos \left(k_{z_{2}} h\right)} e^{-j k_{t} \rho} k_{t} d k_{t}
\end{gathered}
$$

With the integrals put into the above form, it is possible to close an expanding semicircular contour around the poles (zeros of $D_{T E}$ or $D_{T M}$ ) in the lower complex $k_{t}$ plane. Complications arise in the proper definition of $k_{z_{1}}$ :

$$
k_{z_{1}}=\sqrt{k_{0}^{2}-k_{t}^{2}}
$$

which cannot be single-valued and continuous throughout the entire complex $k_{t}$ plane. The lines across which this function is discontinuous, the branch cuts, depend on the choice of the positive or negative root in various regions of the complex $k_{t}$ plane. The algebraic form of Sommerfeld's choice for $k_{z_{1}}$ is discussed in Appendix $\mathrm{B}$ and is given by

$$
k_{z_{1}} \equiv-\sqrt{\left|k_{0}{ }^{2}-k_{t}{ }^{2}\right|} \exp \left[\frac{j}{2} \arg _{0}\left(k_{0}{ }^{2}-k_{t}{ }^{2}\right)\right]
$$

where $\arg _{0}\left(k_{0}{ }^{2}-k_{t}{ }^{2}\right)$ is the value of $\arg \left(k_{0}{ }^{2}-k_{t}{ }^{2}\right)$ that lies between 0 and $2 \pi$. The branch cuts and behavior of $k_{z_{1}}$ for this choice are depicted in the complex $k_{t}$ plane in Fig. 2. 


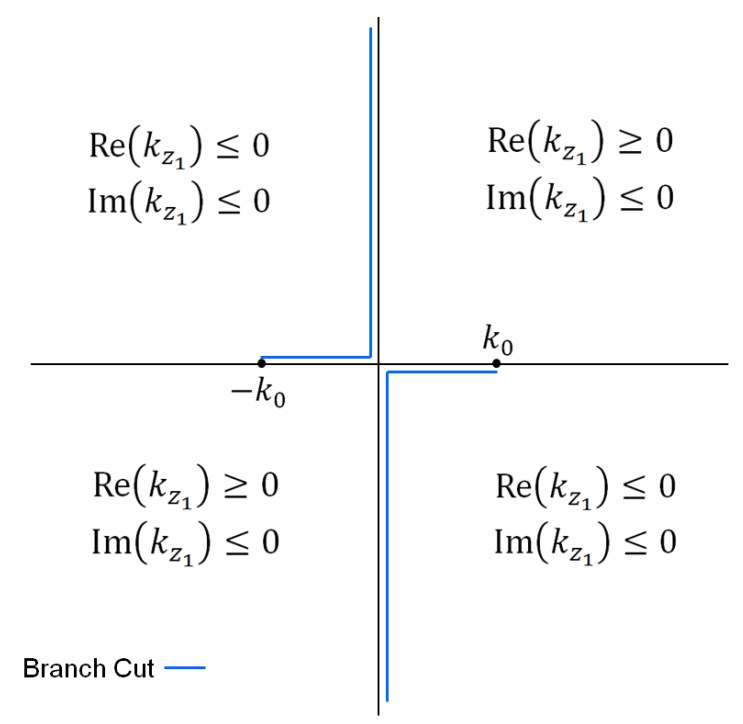

Fig. 2 Branch cuts and behavior of $k_{z_{1}}$ for this choice in the complex $k_{t}$ plane

On the real axis, just above the branch cuts, this definition reduces to the usual requirement that waves be attenuated exponentially:

$$
\begin{gathered}
k_{z_{1}}=\sqrt{k_{0}{ }^{2}-k_{t}^{2}} \quad, \quad k_{t}<k_{0} \\
k_{z_{1}}=-j \sqrt{{k_{t}{ }^{2}-k_{0}^{2}}^{2}} \quad, \quad k_{t}>k_{0}
\end{gathered}
$$

Throughout the rest of the complex plane, Eq. 56 preserves the property that $\operatorname{Im}\left(k_{z_{1}}\right)<0$. The closed contour that we use is shown in Fig. 3.

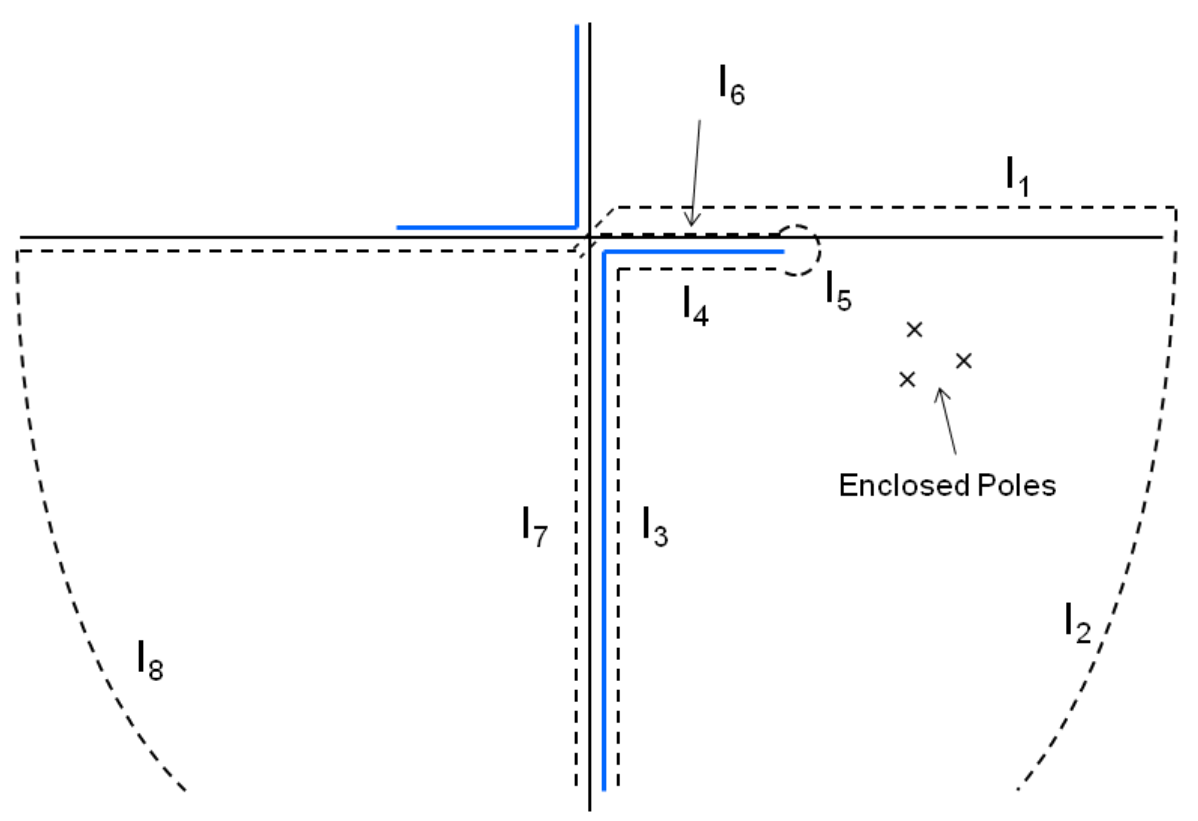

Fig. 3 The closed contour that we use 
By the residue theorem, we have

$$
I_{1}+I_{2}+I_{3}+I_{4}+I_{5}+I_{6}+I_{7}+I_{8}=-2 \pi j \sum \operatorname{res}\left(k_{p}\right)
$$

Since the semicircular arc is expanding into a proper region, Jordan's Lemma says that this integral will go to 0 as the radius of this arc goes to infinity:

$$
I_{2}+I_{8} \rightarrow 0
$$

Additionally, the integration around the branch point, $I_{5}$, vanishes. Therefore,

$$
I_{1}=-\left(I_{3}+I_{7}\right)-\left(I_{4}+I_{6}\right)-2 \pi j \sum \operatorname{res}\left(k_{p}\right)
$$

where $\left(I_{3}+I_{7}\right)$ and $\left(I_{4}+I_{6}\right)$ do not vanish, since $k_{z_{1}}$ is discontinuous across the branch cuts. An asymptotic analysis of the integration around the branch cut by the method of steepest descent gives a result for the radiated field that, under certain conditions, is in agreement with our earlier results obtained by the method of stationary phase. Thus, Eq. 59 may be interpreted physically as a decomposition of the field into radiated and surface wave components.

In general, the locations of the zeros of either $D_{T E}$ or $D_{T M}$ must be found by a root-finding algorithm on a computer. However, analysis of their expressions indicates that our contour will capture these points in quadrant IV of the complex $k_{t}$ plane. Furthermore, the real part of these zeros will be located somewhere in the interval $\left(k_{0}, \sqrt{\mu_{r} \varepsilon_{r}} k_{0}\right)$. In the limit of a lossless substrate, $\mu_{r}$ and $\varepsilon_{r}$ are real numbers, and the zeros migrate up to the real axis of the $k_{t}$ plane. In this limit, it should actually be understood that the zero is "slightly" below the real axis, so that the formula Eq. 59 still applies. Otherwise, the formula Eq. 59 would be a discontinuous function of the substrate properties in passing from a small amount of loss to no loss.

Using the expression for $H_{z_{1}}$, the form of a $T E^{z}$ surface wave above the substrate is found by calculating the residue at any zero of $D_{T E}$ :

$$
\begin{gathered}
H_{z_{1}}{ }^{S W}=\frac{\ell I_{0} \mu_{r} \sin \phi}{2} e^{j \frac{\pi}{4}} \lim _{k_{t} \rightarrow k_{p}} k_{t}-k_{p} \frac{\sqrt{\frac{2 k_{t}}{\pi \rho}} e^{-j k_{z_{1}} z} e^{-j k_{t} \rho} k_{t}}{D_{T E}} \\
H_{z_{1}}{ }^{S W}=\frac{\ell I_{0} \mu_{r} \sin \phi}{2} e^{j \frac{\pi}{4}} \sqrt{\frac{2 k_{p}}{\pi \rho}} e^{-j k_{z_{1}} z} e^{-j k_{p} \rho} k_{p} \lim _{k_{t} \rightarrow k_{p}} \frac{1}{\frac{D_{T E}\left(k_{t}\right)-D_{T E}\left(k_{p}\right)}{k_{t}-k_{p}}} \\
H_{z_{1}}{ }^{S W}=\frac{\ell I_{0} \mu_{r} \sin \phi}{2} e^{j \frac{\pi}{4}} \sqrt{\frac{2 k_{p}}{\pi \rho}} e^{-j k_{z_{1}} z} e^{-j k_{p} \rho} k_{p} \frac{1}{D_{T E}^{\prime}\left(k_{p}\right)}
\end{gathered}
$$


where $D_{T E}{ }^{\prime}\left(k_{p}\right)$ is the derivative of $D_{T E}$ with respect to $k_{t}$ evaluated at the pole location $k_{p} . k_{z_{1}}$ is also evaluated at the pole location. By the same process, we can write the expressions for the z-components of the surface wave fields:

$$
\begin{gathered}
\underline{T E^{z} \text { Surface Wave }} \\
H_{z_{1}}{ }^{S W}=\frac{\ell I_{0} \mu_{r} \sin \phi}{2} e^{j \frac{\pi}{4}} \sqrt{\frac{2 k_{p}}{\pi \rho} e^{-j k_{z_{1}} z} e^{-j k_{p} \rho} k_{p} \frac{1}{D_{T E}{ }^{\prime}\left(k_{p}\right)}} \\
H_{z_{2}}{ }^{S W}=\frac{\ell I_{0} \sin \phi}{2} e^{j \frac{\pi}{4}} \sqrt{\frac{2 k_{p}}{\pi \rho}} \frac{\sin \left[k_{z_{2}}(z+h)\right]}{\sin \left(k_{z_{2}} h\right)} e^{-j k_{p} \rho} k_{p} \frac{1}{D_{T E}{ }^{\prime}\left(k_{p}\right)} \\
E_{z_{1}} S W=\frac{j \ell I_{0} \eta_{0} \cos \phi}{2 k_{0}} e^{j \frac{\pi}{4}} \sqrt{\frac{2 k_{p}}{\pi \rho} \tan \left(k_{z_{2}} h\right) e^{-j k_{z_{1}}{ }^{z}} e^{-j k_{p} \rho} k_{z_{2}} k_{p} \frac{1}{D_{T M}{ }^{\prime}\left(k_{p}\right)}} \\
E_{z_{2}}{ }^{S W}=-\frac{\ell I_{0} \eta_{0} \cos \phi}{2 k_{0}} e^{j \frac{\pi}{4}} \sqrt{\frac{2 k_{p}}{\pi \rho}} \frac{\cos \left[k_{z_{2}}(z+h)\right]}{\cos \left(k_{z_{2}} h\right)} e^{-j k_{p} \rho} k_{z_{1}} k_{p} \frac{1}{D_{T M}{ }^{\prime}\left(k_{p}\right)}
\end{gathered}
$$

\section{Radiated Power, Directivity, and Efficiency}

The Poynting vector describing the power flow associated with the far-field radiation is given by the following formula:

$$
\boldsymbol{S}_{\text {avg }}=\frac{1}{2 \eta_{0}} \operatorname{Re}\left(\boldsymbol{E} \cdot \boldsymbol{E}^{*}\right)=\hat{\boldsymbol{r}} \frac{1}{2}\left(\left|E_{\theta}\right|^{2}+\left|E_{\phi}\right|^{2}\right)
$$

The expressions for $E_{\theta}$ and $E_{\phi}$ were given in Eq. 47. They are repeated below:

$$
\begin{gathered}
E_{\theta}=\frac{\ell I_{0} \eta_{0} j k_{x_{s}} k_{z_{2_{s}}} \cot \theta}{k_{0} D_{T M_{s}}} \tan \left(k_{z_{2_{s}}} h\right) \frac{e^{-j k_{0} r}}{2 \pi r} \\
E_{\phi}=-\frac{\ell I_{0} \eta_{0} \mu_{r} k_{y_{S}} \cot \theta}{D_{T E_{S}}} \frac{e^{-j k_{0} r}}{2 \pi r}
\end{gathered}
$$

Combining Eqs. 62 and 63, we have 


$$
\boldsymbol{S}_{\text {avg }}=\frac{\ell^{2} I_{0}{ }^{2} \eta_{0}}{8 \pi^{2} r^{2}}\left(\left|\frac{k_{x_{s}} k_{z_{2_{S}}} \cot \theta}{k_{0} D_{T M_{s}}} \tan \left(k_{z_{2_{s}}} h\right)\right|^{2}+\left|\frac{\mu_{r} k_{y_{s}} \cot \theta}{D_{T E_{s}}}\right|^{2}\right)
$$

The total power radiated is found by integrating the Poynting vector over the surface of a hemisphere of radius $r$ :

$$
\begin{aligned}
& P_{\text {rad }}=\frac{\ell^{2} I_{0}{ }^{2} \eta_{0}}{8 \pi^{2} r^{2}} \int_{0}^{2 \pi} \int_{0}^{\pi / 2}\left(\left|\frac{k_{x_{s}} k_{z_{2_{S}}} \cot \theta}{k_{0} D_{T M_{S}}} \tan \left(k_{z_{2_{S}}} h\right)\right|^{2}+\left|\frac{\mu_{r} k_{y_{s}} \cot \theta}{D_{T E_{S}}}\right|^{2}\right) r^{2} \sin \theta d \theta d \phi \\
& P_{\text {rad }}=\frac{\ell^{2} I_{0}^{2} \eta_{0}}{8 \pi^{2}} \int_{0}^{2 \pi} \int_{0}^{\pi / 2}\left(\left|\frac{k_{x_{s}} k_{z_{2_{S}}} \cot \theta}{k_{0} D_{T M_{S}}} \tan \left(k_{z_{z_{s}}} h\right)\right|^{2}+\left|\frac{\mu_{r} k_{y_{s}} \cot \theta}{D_{T E_{S}}}\right|^{2}\right) \sin \theta d \theta d \phi \\
& P_{\text {rad }}=\frac{\ell^{2} I_{0}{ }^{2} \eta_{0}}{8 \pi^{2}} \int_{0}^{2 \pi} \int_{0}^{\pi / 2}\left(\left|\frac{k_{0} \sin \theta \cos \phi k_{z_{z_{s}}} \cot \theta}{k_{0} D_{T M_{s}}} \tan \left(k_{z_{z_{s}}} h\right)\right|^{2}\right. \\
& \left.+\left|\frac{\mu_{r} k_{0} \sin \theta \sin \phi \cot \theta}{D_{T E_{s}}}\right|^{2}\right) \sin \theta d \theta d \phi \\
& P_{\text {rad }}=\frac{\ell^{2} I_{0}{ }^{2} \eta_{0}}{8 \pi^{2}} \int_{0}^{2 \pi} \int_{0}^{\pi / 2}\left(\left|\frac{k_{0} \cos \phi k_{z_{s}} \cos \theta}{k_{0} D_{T M_{s}}} \tan \left(k_{z_{z_{S}}} h\right)\right|^{2}+\left|\frac{\mu_{r} k_{0} \sin \phi \cos \theta}{D_{T E_{s}}}\right|^{2}\right) \sin \theta d \theta d \phi \\
& P_{\text {rad }}=\frac{\ell^{2} I_{0}{ }^{2} \eta_{0}}{8 \pi} \int_{0}^{\pi / 2}\left(\left|\frac{k_{0} k_{z_{2_{S}}} \cos \theta}{k_{0} D_{T M_{S}}} \tan \left(k_{z_{2_{S}}} h\right)\right|^{2}+\left|\frac{\mu_{r} k_{0} \cos \theta}{D_{T E_{S}}}\right|^{2}\right) \sin \theta d \theta \\
& P_{\text {rad }}=\frac{k_{0}^{2} \ell^{2} I_{0}{ }^{2} \eta_{0}}{8 \pi} \int_{0}^{\pi / 2}\left(\left|\frac{k_{z_{2_{s}}} \tan \left(k_{z_{2_{s}}} h\right)}{k_{0} D_{T M_{s}}}\right|^{2}+\left|\frac{\mu_{r}}{D_{T E_{s}}}\right|^{2}\right) \cos ^{2} \theta \sin \theta d \theta
\end{aligned}
$$

The last integration over $\theta$ cannot be done analytically. The directivity is

$$
D(\theta, \phi)=\frac{4 \pi r^{2}\left|\boldsymbol{S}_{\text {avg }}\right|}{P_{\text {rad }}}
$$

Furthermore, we can define the radiation resistance in the usual way:

$$
R_{r}=\frac{2 P_{r a d}}{I_{0}{ }^{2}}
$$

We would also like to calculate the power launched into any excited surface waves. If only 1 surface wave is excited, then it is feasible to use the expressions for the surface wave fields derived in the previous section to determine the power. However, a more general procedure that 
accounts for any power launched into surface waves is used here. Consider the Poynting theorem for lossless media:

$$
-\frac{1}{2} \iiint \boldsymbol{E} \cdot \boldsymbol{J}^{*} d V=\oiint \boldsymbol{S}_{\boldsymbol{a v g}} \cdot \boldsymbol{d} \boldsymbol{S}+j \frac{\omega}{2} \iiint\left(\mu|\boldsymbol{H}|^{2}-\varepsilon|\boldsymbol{E}|^{2}\right) d V
$$

If we take the real part of both sides of Eq. 68, then we obtain

$$
P_{\text {total }}=\oiint \boldsymbol{S}_{\boldsymbol{a v g}} \cdot \boldsymbol{d} \boldsymbol{S}=-\frac{1}{2} \operatorname{Re} \iiint \boldsymbol{E} \cdot \boldsymbol{J}^{*} d V
$$

In Eq. 69, we take $\boldsymbol{S}_{\boldsymbol{a v g}}$ to be the total power flow produced by the source. Thus, $\boldsymbol{S}_{\boldsymbol{a v g}}$ in Eq. 69 is not the same $\boldsymbol{S}_{\boldsymbol{a v g}}$ that was used in Eq. 64 to calculate the power radiated into the upper hemisphere. Working with Eq. 69,

$$
\begin{gathered}
P_{\text {total }}=-\frac{1}{2} \operatorname{Re} \iiint_{\text {total }}=-\left.\frac{1}{2} \operatorname{Re} \iint_{-\infty}^{\infty} E_{x}\right|_{z=0} \ell I_{0} \delta(x) \delta(y) d x d y \\
\left.P_{0} \delta(x) \delta(y) \delta(z)\right) d V
\end{gathered}
$$

By Parseval's theorem, Eq. 70 is equivalent to

$$
P_{\text {total }}=-\left.\frac{1}{8 \pi^{2}} \operatorname{Re} \iint_{-\infty}^{\infty} \widetilde{E_{x}}\right|_{z=0} \ell I_{0} d k_{x} d k_{y}
$$

In order to use Eq. 71, we have to detour slightly to derive a convenient expression for $\widetilde{E_{x}} . \widetilde{E_{x}}$ may be found from the vector potential as follows:

$$
\left.\widetilde{E_{x}}\right|_{z=0}=\left.\frac{1}{j \omega \mu \varepsilon}\left[-j k_{x} \frac{\partial \widetilde{A_{z_{1}}}}{\partial z}+\left(k_{z_{1}}{ }^{2}+k_{y}{ }^{2}\right) \widetilde{A_{x_{1}}}\right]\right|_{z=0^{+}}
$$

From Eq. 29, we have

$$
\begin{gathered}
\widetilde{A_{x_{1}}}=\frac{\mu_{0} \mu_{r} \ell I_{0}}{D_{T E}} e^{-j k_{z_{1}} z} \\
\widetilde{A_{z_{1}}}=\frac{-\mu_{0} \ell I_{0} j k_{x}\left(\mu_{r} \varepsilon_{r}-1\right)}{D_{T E} D_{T M}} e^{-j k_{z_{1}} z}
\end{gathered}
$$

Combining Eqs. 72 and 73, we have

$$
\left.\widetilde{E_{x}}\right|_{z=0}=\ell I_{0} \eta_{0}\left[\frac{k_{x}{ }^{2} k_{z_{1}}\left(\mu_{r} \varepsilon_{r}-1\right)}{k_{0} D_{T E} D_{T M}}-\frac{j \mu_{r}\left(k_{z_{1}}{ }^{2}+k_{y}{ }^{2}\right)}{k_{0} D_{T E}}\right]
$$

Equation 74 can be manipulated into a form more convenient for the integration that follows. We begin by substituting Eq. 34 into Eq. 74, yielding 


$$
\left.\widetilde{E_{x}}\right|_{z=0}=\ell I_{0} \eta_{0}\left[\frac{k_{x}{ }^{2} k_{z_{1}}\left({k_{z_{2}}}^{2}-\mu_{r} \varepsilon_{r}{k_{z_{1}}}^{2}\right)}{\left(k_{x}{ }^{2}+k_{y}{ }^{2}\right) k_{0} D_{T E} D_{T M}}-\frac{j \mu_{r}\left({k_{z_{1}}}^{2}+k_{y}{ }^{2}\right)}{k_{0} D_{T E}}\right]
$$

From the definition of $D_{T M}$, we have

$$
\varepsilon_{r} k_{z_{1}}=-j\left(D_{T M}+k_{z_{2}} \tan \left(k_{z_{2}} h\right)\right)
$$

Combining Eqs. 75 and 76,

$$
\begin{aligned}
& \left.\widetilde{E_{x}}\right|_{z=0}=\ell I_{0} \eta_{0}\left[\frac{k_{x}{ }^{2} k_{z_{1}}\left[k_{z_{2}}{ }^{2}+j \mu_{r} k_{z_{1}}\left(D_{T M}+k_{z_{2}} \tan \left(k_{z_{2}} h\right)\right)\right]}{\left(k_{x}{ }^{2}+k_{y}{ }^{2}\right) k_{0} D_{T E} D_{T M}}-\frac{j \mu_{r}\left(k_{z_{1}}{ }^{2}+k_{y}{ }^{2}\right)}{k_{0} D_{T E}}\right] \\
& \left.\widetilde{E_{x}}\right|_{z=0}=\ell I_{0} \eta_{0}\left[\frac{k_{x}{ }^{2} k_{z_{1}}\left[k_{z_{2}}{ }^{2}+j \mu_{r} k_{z_{1}} k_{z_{2}} \tan \left(k_{z_{2}} h\right)+j \mu_{r} k_{z_{1}} D_{T M}\right]}{\left(k_{x}{ }^{2}+k_{y}{ }^{2}\right) k_{0} D_{T E} D_{T M}}-\frac{j \mu_{r}\left(k_{z_{1}}{ }^{2}+k_{y}{ }^{2}\right)}{k_{0} D_{T E}}\right] \\
& \left.\widetilde{E_{x}}\right|_{z=0}=\ell I_{0} \eta_{0}\left[\frac{k_{x}{ }^{2} k_{z_{1}}\left[{k_{z_{2}}}^{2}+j \mu_{r} k_{z_{1}} k_{z_{2}} \tan \left(k_{z_{2}} h\right)\right]}{\left(k_{x}{ }^{2}+k_{y}{ }^{2}\right) k_{0} D_{T E} D_{T M}}+\frac{j \mu_{r} k_{x}{ }^{2} k_{z_{1}}{ }^{2}}{\left(k_{x}{ }^{2}+k_{y}{ }^{2}\right) k_{0} D_{T E}}\right. \\
& \left.-\frac{j \mu_{r}\left(k_{z_{1}}{ }^{2}+k_{y}{ }^{2}\right)}{k_{0} D_{T E}}\right] \\
& \left.\widetilde{E_{x}}\right|_{z=0}=\ell I_{0} \eta_{0}\left[\frac{k_{x}{ }^{2}{k_{z_{1}}}\left[{k_{z_{2}}}^{2}+j \mu_{r} k_{z_{1}} k_{z_{2}} \tan \left(k_{z_{2}} h\right)\right] \cot \left(k_{z_{2}} h\right)}{\left(k_{x}{ }^{2}+k_{y}{ }^{2}\right) k_{0} D_{T E} D_{T M} \cot \left(k_{z_{2}} h\right)}+\frac{j \mu_{r} k_{x}{ }^{2}{k_{z_{1}}}^{2}}{\left(k_{x}{ }^{2}+k_{y}{ }^{2}\right) k_{0} D_{T E}}\right. \\
& \left.-\frac{j \mu_{r}\left(k_{z_{1}}{ }^{2}+k_{y}^{2}\right)}{k_{0} D_{T E}}\right] \\
& \left.\widetilde{E_{x}}\right|_{z=0}=\ell I_{0} \eta_{0}\left[\frac{k_{x}{ }^{2} k_{z_{1}} k_{z_{2}}\left[k_{z_{2}} \cot \left(k_{z_{2}} h\right)+j \mu_{r} k_{z_{1}}\right]}{\left(k_{x}{ }^{2}+k_{y}{ }^{2}\right) k_{0} D_{T E} D_{T M} \cot \left(k_{z_{2}} h\right)}+\frac{j \mu_{r} k_{x}{ }^{2} k_{z_{1}}{ }^{2}}{\left(k_{x}{ }^{2}+k_{y}{ }^{2}\right) k_{0} D_{T E}}-\frac{j \mu_{r}\left(k_{z_{1}}{ }^{2}+k_{y}{ }^{2}\right)}{k_{0} D_{T E}}\right] \\
& \left.\widetilde{E_{x}}\right|_{z=0}=\ell I_{0} \eta_{0}\left[\frac{k_{x}{ }^{2} k_{z_{1}} k_{z_{2}}\left[D_{T E}\right]}{\left(k_{x}{ }^{2}+k_{y}{ }^{2}\right) k_{0} D_{T E} D_{T M} \cot \left(k_{z_{2}} h\right)}+\frac{j \mu_{r} k_{x}{ }^{2} k_{z_{1}}{ }^{2}}{\left(k_{x}{ }^{2}+k_{y}{ }^{2}\right) k_{0} D_{T E}}-\frac{j \mu_{r}\left(k_{z_{1}}{ }^{2}+k_{y}{ }^{2}\right)}{k_{0} D_{T E}}\right] \\
& \left.\widetilde{E_{x}}\right|_{z=0}=\ell I_{0} \eta_{0}\left[\frac{k_{x}{ }^{2} k_{z_{1}} k_{z_{2}} \tan \left(k_{z_{2}} h\right)}{k_{0}\left(k_{x}{ }^{2}+k_{y}{ }^{2}\right) D_{T M}}+\frac{j \mu_{r} k_{x}{ }^{2}{k_{z_{1}}}^{2}-j \mu_{r}\left(k_{x}{ }^{2}+k_{y}{ }^{2}\right)\left({k_{z_{1}}}^{2}+k_{y}{ }^{2}\right)}{\left(k_{x}{ }^{2}+k_{y}{ }^{2}\right) k_{0} D_{T E}}\right] \\
& \left.\widetilde{E_{x}}\right|_{z=0}=\ell I_{0} \eta_{0}\left[\frac{k_{x}{ }^{2} k_{z_{1}} k_{z_{2}} \tan \left(k_{z_{2}} h\right)}{k_{0}\left(k_{x}{ }^{2}+k_{y}{ }^{2}\right) D_{T M}}+\frac{j \mu_{r} k_{x}{ }^{2}{k_{z_{1}}}^{2}-j \mu_{r}\left(k_{x}{ }^{2}+k_{y}{ }^{2}\right)\left(k_{z_{1}}{ }^{2}+k_{y}{ }^{2}\right)}{\left(k_{x}{ }^{2}+k_{y}{ }^{2}\right) k_{0} D_{T E}}\right] \\
& \left.\widetilde{E_{x}}\right|_{z=0}=\ell I_{0} \eta_{0}\left[\frac{k_{x}{ }^{2} k_{z_{1}} k_{z_{2}} \tan \left(k_{z_{2}} h\right)}{k_{0}\left(k_{x}{ }^{2}+k_{y}{ }^{2}\right) D_{T M}}+\frac{j \mu_{r}\left(k_{x}{ }^{2} k_{z_{1}}{ }^{2}-k_{x}{ }^{2}{k_{z_{1}}}^{2}-k_{y}{ }^{2}{k_{z_{1}}}{ }^{2}-k_{x}{ }^{2} k_{y}{ }^{2}-k_{y}{ }^{4}\right)}{\left(k_{x}{ }^{2}+k_{y}{ }^{2}\right) k_{0} D_{T E}}\right] \\
& \left.\widetilde{E_{x}}\right|_{z=0}=\ell I_{0} \eta_{0}\left[\frac{k_{x}{ }^{2} k_{z_{1}} k_{z_{2}} \tan \left(k_{z_{2}} h\right)}{k_{0}\left(k_{x}{ }^{2}+k_{y}{ }^{2}\right) D_{T M}}-\frac{j \mu_{r} k_{y}{ }^{2}\left(k_{z_{1}}{ }^{2}+k_{x}{ }^{2}+k_{y}{ }^{2}\right)}{\left(k_{x}{ }^{2}+k_{y}{ }^{2}\right) k_{0} D_{T E}}\right] \\
& \left.\widetilde{E_{x}}\right|_{z=0}=\ell I_{0} \eta_{0}\left[\frac{k_{x}{ }^{2} k_{z_{1}} k_{z_{2}} \tan \left(k_{z_{2}} h\right)}{k_{0}\left(k_{x}{ }^{2}+k_{y}{ }^{2}\right) D_{T M}}-\frac{j \mu_{r} k_{y}{ }^{2}\left(k_{0}{ }^{2}\right)}{\left(k_{x}{ }^{2}+k_{y}{ }^{2}\right) k_{0} D_{T E}}\right]
\end{aligned}
$$




$$
\left.\widetilde{E_{x}}\right|_{z=0}=\ell I_{0} \eta_{0}\left[\frac{k_{x}{ }^{2} k_{z_{1}} k_{z_{2}} \tan \left(k_{z_{2}} h\right)}{k_{0}\left(k_{x}{ }^{2}+k_{y}{ }^{2}\right) D_{T M}}-\frac{j \mu_{r} k_{y}{ }^{2} k_{0}}{\left(k_{x}{ }^{2}+k_{y}{ }^{2}\right) D_{T E}}\right]
$$

Substitution of Eq. 77 into Eq. 71 gives

$$
P_{\text {total }}=-\frac{\ell^{2} I_{0}{ }^{2} \eta_{0}}{8 \pi^{2}} \operatorname{Re} \iint_{-\infty}^{\infty}\left[\frac{k_{x}{ }^{2} k_{z_{1}} k_{z_{2}} \tan \left(k_{z_{2}} h\right)}{k_{0}\left(k_{x}{ }^{2}+k_{y}{ }^{2}\right) D_{T M}}-\frac{j \mu_{r} k_{y}{ }^{2} k_{0}}{\left(k_{x}{ }^{2}+k_{y}{ }^{2}\right) D_{T E}}\right] d k_{x} d k_{y}
$$

We then introduce the change of variables into Eq. 78:

$$
\begin{aligned}
& k_{x}=k_{t} \cos \alpha \\
& k_{y}=k_{t} \sin \alpha
\end{aligned}
$$

yielding

$$
\begin{gathered}
P_{\text {total }}=-\frac{\ell^{2} I_{0}{ }^{2} \eta_{0}}{8 \pi^{2}} \operatorname{Re} \int_{0}^{2 \pi} \int_{0}^{\infty}\left[\frac{\cos ^{2} \alpha k_{z_{1}} k_{z_{2}} \tan \left(k_{z_{2}} h\right)}{k_{0} D_{T M}}-\frac{j \mu_{r} k_{0} \sin ^{2} \alpha}{D_{T E}}\right] k_{t} d k_{t} d \alpha \\
P_{\text {total }}=-\frac{\ell^{2} I_{0}{ }^{2} \eta_{0}}{8 \pi} \operatorname{Re} \int_{0}^{\infty}\left[\frac{k_{z_{1}} k_{z_{2}} \tan \left(k_{z_{2}} h\right)}{k_{0} D_{T M}}-\frac{j \mu_{r} k_{0}}{D_{T E}}\right] k_{t} d k_{t}
\end{gathered}
$$

In the limit of a lossless substrate, poles exist along the integration interval of Eq. 80, thus this integral must be interpreted in terms of Cauchy principal value integrals. This is similar to what was done earlier in the analysis of the surface wave fields. If we interpret Eq. 80 as a contour integral along the real axis of the complex $k_{t}$ plane, and we adopt the same definition for $k_{z_{1}}$, then we may integrate along any path so long as we start and stop at the same points, since all the functions in the integrand are analytic. Consider the contour shown in Fig. 4, where the integration path avoids the difficulties associated with the poles by integrating around them. ${ }^{4}$

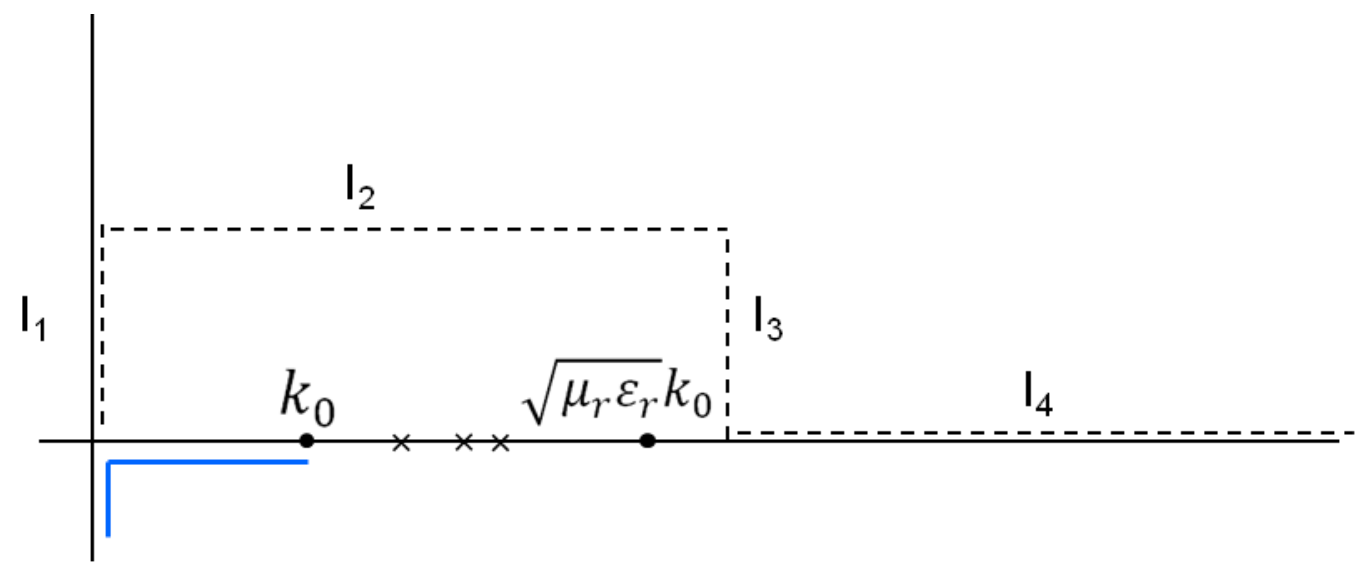

Fig. 4 Alternative path of integration 
We have

$$
P_{\text {total }}=\operatorname{Re}\left(I_{1}+I_{2}+I_{3}+I_{4}\right)
$$

However, the integrand for $I_{4}$ is pure imaginary so long as the starting point is past $\sqrt{\mu_{r} \varepsilon_{r}} k_{0}$, so that the total power can be found by numerically integrating around the box $I_{1}+I_{2}+I_{3}$. The difference between the total power and the radiated power is the power launched into surface waves. We define

$$
P_{S W}=P_{\text {total }}-P_{\text {rad }}
$$

Additionally, it is worth noting that integrating from 0 to $k_{0}$ gives the power radiated into the upper hemisphere. ${ }^{5}$ Although a proof of this fact is not known to the author, numerical calculations were always in agreement with results obtained from Eq. 65. Lastly, we may define a surface wave resistance and efficiency as

$$
\begin{gathered}
R_{r}=\frac{2 P_{\text {rad }}}{I_{0}{ }^{2}} \\
\% \text { eff }=\frac{P_{\text {rad }}}{P_{\text {total }}}
\end{gathered}
$$

Some numerical results obtained from the formulas in this section are included in Figs. 5-16. Note that wavelengths on the horizontal axis are measured as wavelengths in that material, and not in terms of free space wavelength. In all cases, a dipole whose length is $1 / 50$ of the free space wavelength is assumed.

For $\varepsilon_{r}=1.01 ; \mu_{r}=1.01 ; \ell=\lambda_{0} / 50$ 


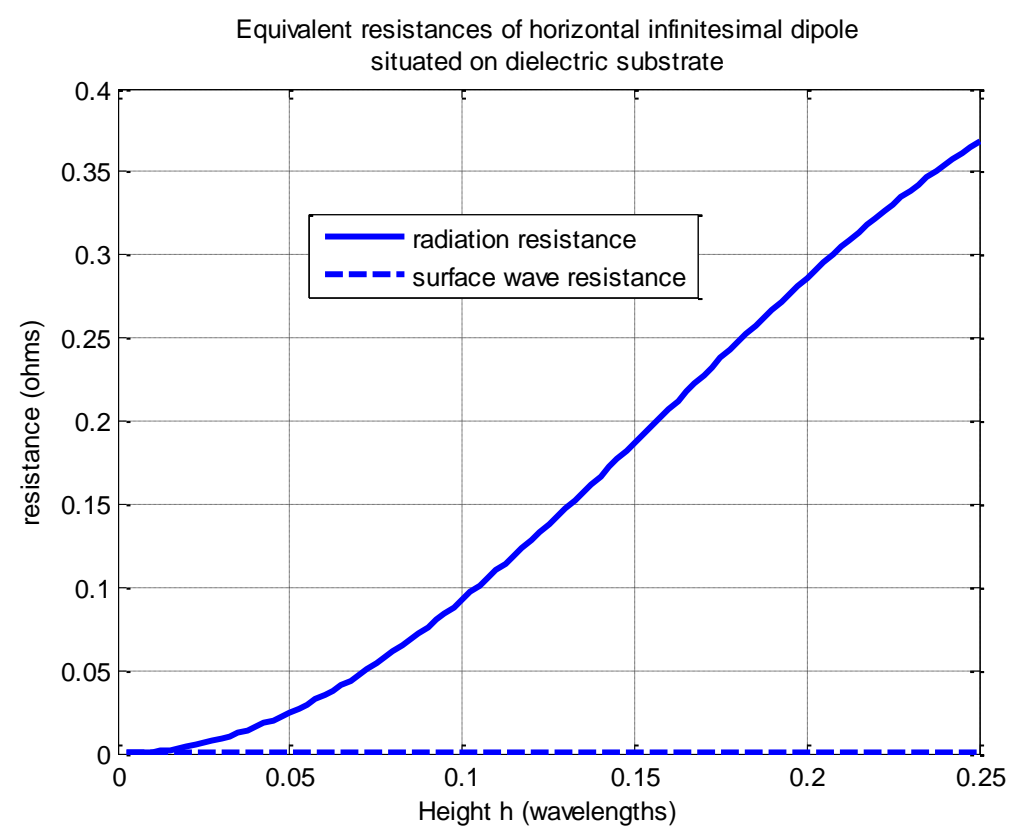

Fig. 5 Equivalent resistances of a horizontal infinitesimal dipole situated on a dielectric substrate; $\varepsilon_{r}=1.01 ; \mu_{r}=1.01 ; \ell=\lambda_{0} / 50$

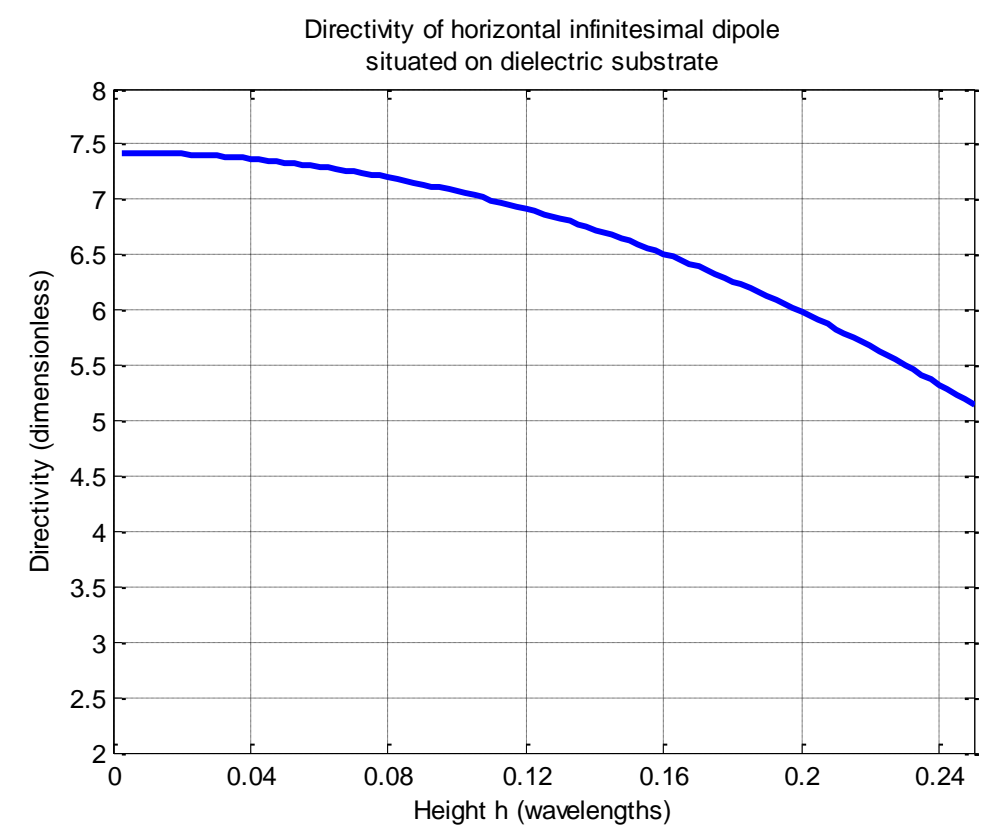

Fig. 6 Directivity of a horizontal dipole situated on a dielectric substrate; $\varepsilon_{r}=1.01 ; \mu_{r}=1.01 ; \ell=\lambda_{0} / 50$ 


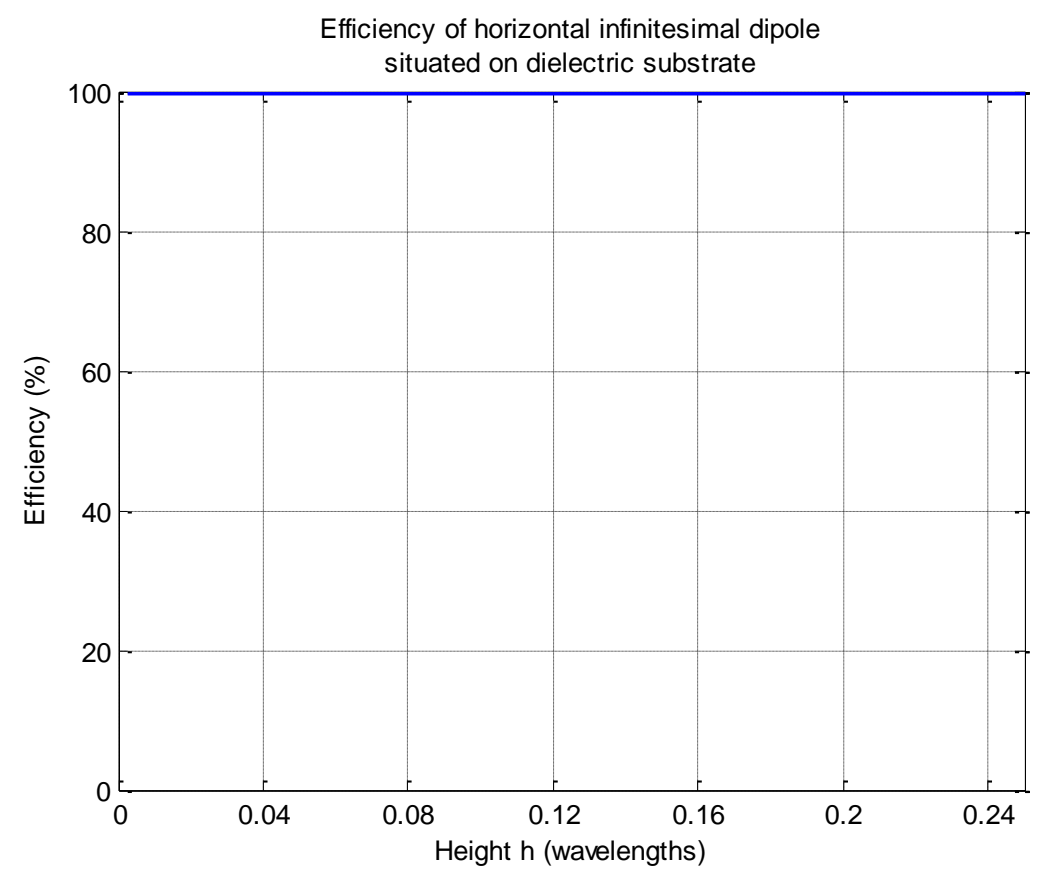

Fig. 7 Efficiency of a horizontal infinitesimal dipole situated on a dielectric substrate; $\varepsilon_{r}=1.01 ; \mu_{r}=1.01 ; \ell=\lambda_{0} / 50$

For $\varepsilon_{r}=10 ; \mu_{r}=1.01 ; \ell=\lambda_{0} / 50$

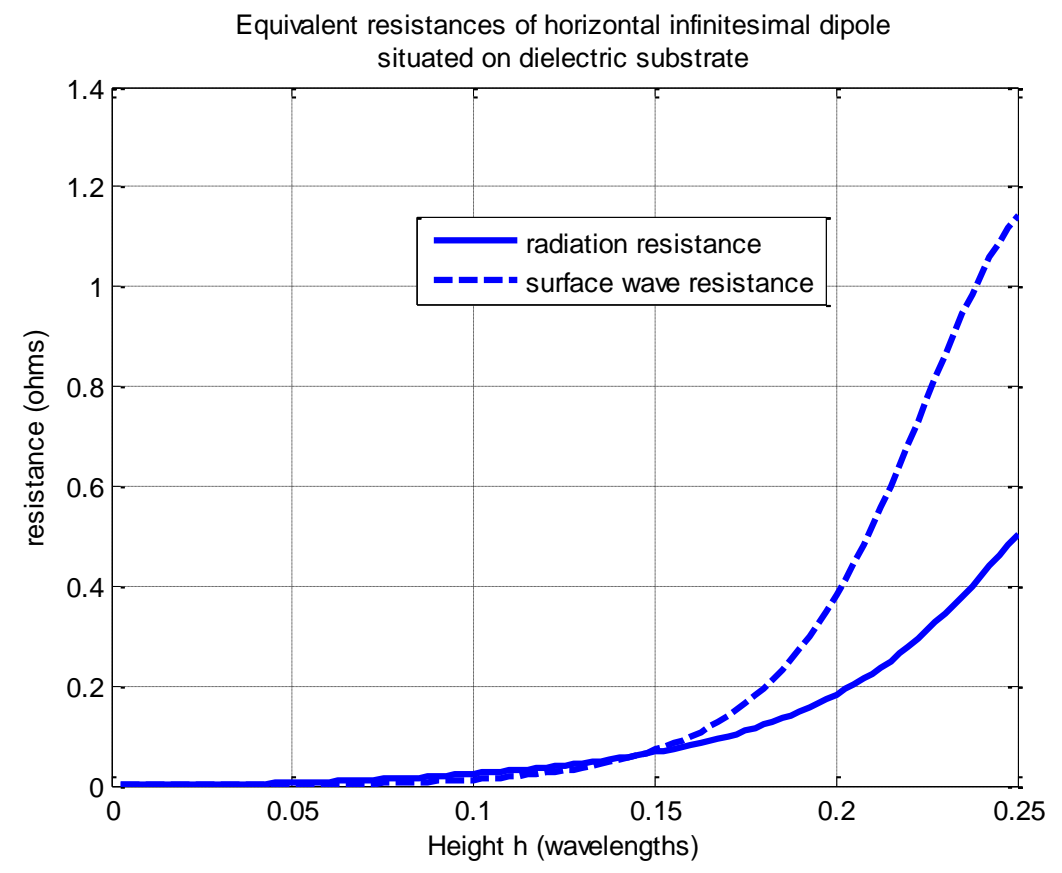

Fig. 8 Equivalent resistances of a horizontal infinitesimal dipole situated on a dielectric substrate; $\varepsilon_{r}=10 ; \mu_{r}=1.01 ; \ell=\lambda_{0} / 50$ 


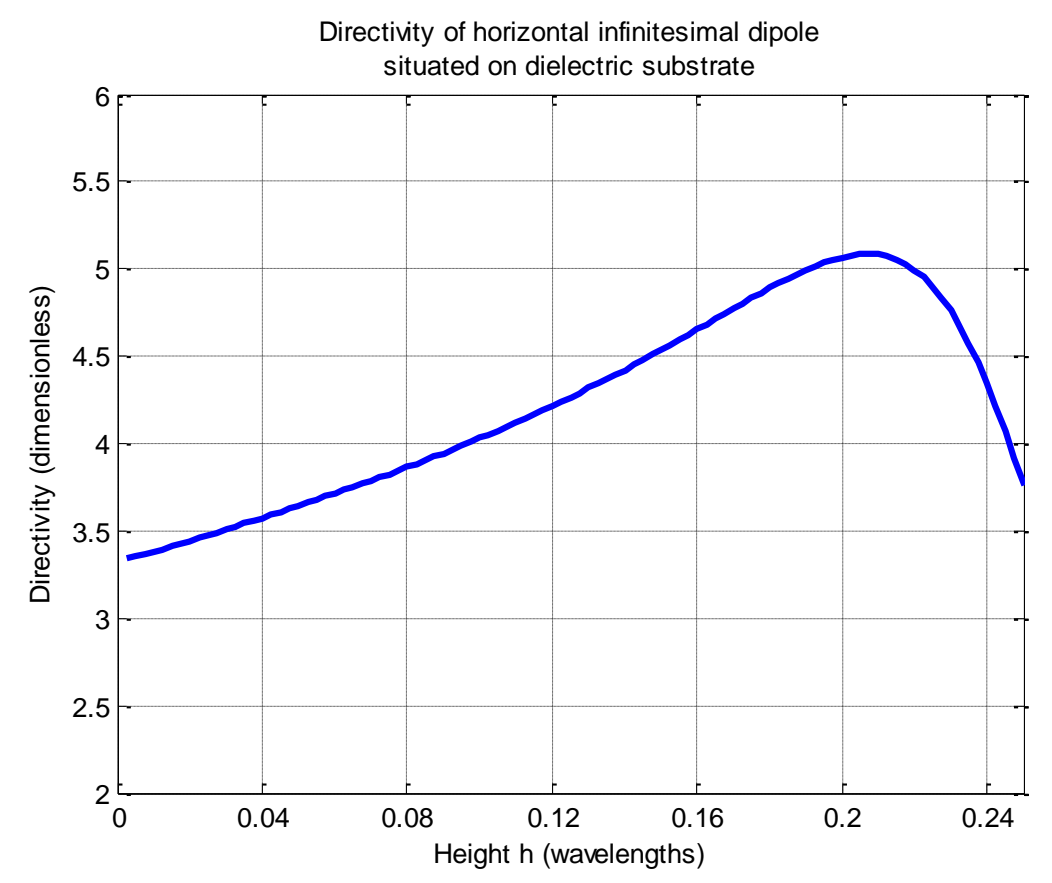

Fig. 9 Directivity of a horizontal infinitesimal dipole situated on a dielectric substrate; $\varepsilon_{r}=10 ; \mu_{r}=1.01 ; \ell=\lambda_{0} / 50$

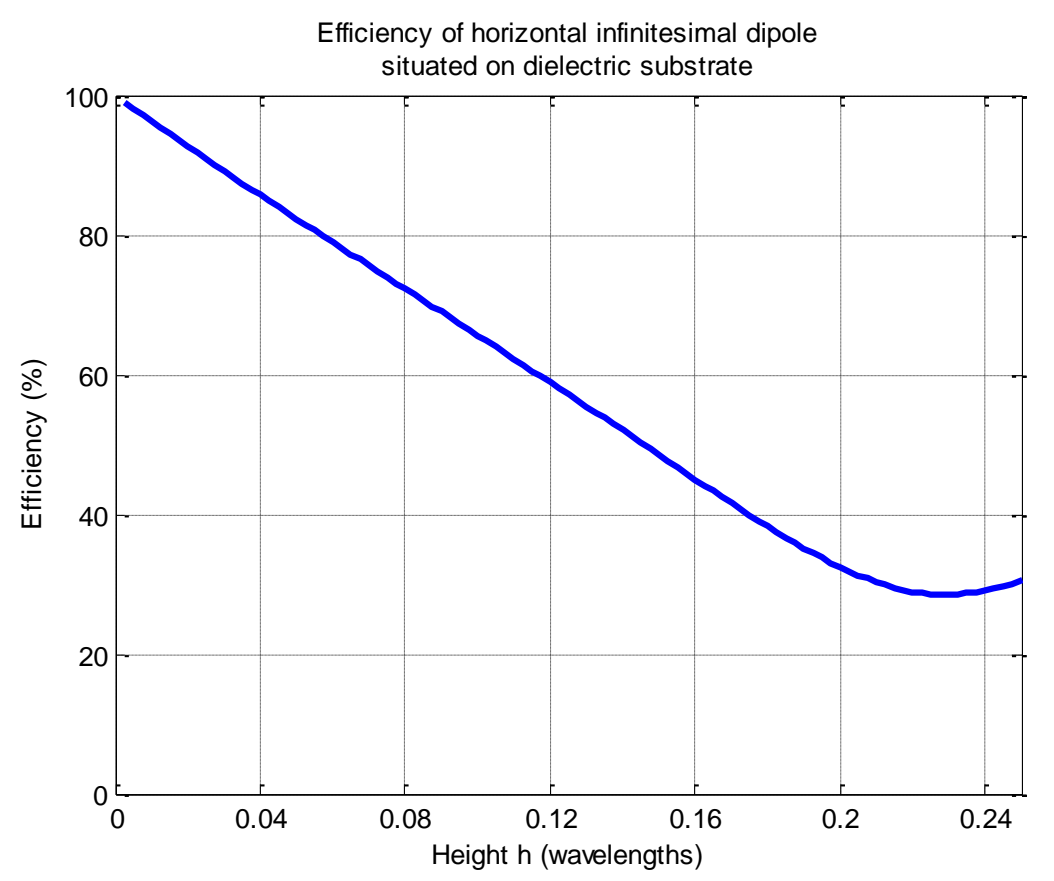

Fig. 10 Efficiency of a horizontal infinitesimal dipole situated on a dielectric substrate; $\varepsilon_{r}=10 ; \mu_{r}=1.01 ; \ell=\lambda_{0} / 50$

For $\varepsilon_{r}=1.01 ; \mu_{r}=10 ; \ell=\lambda_{0} / 50$ 


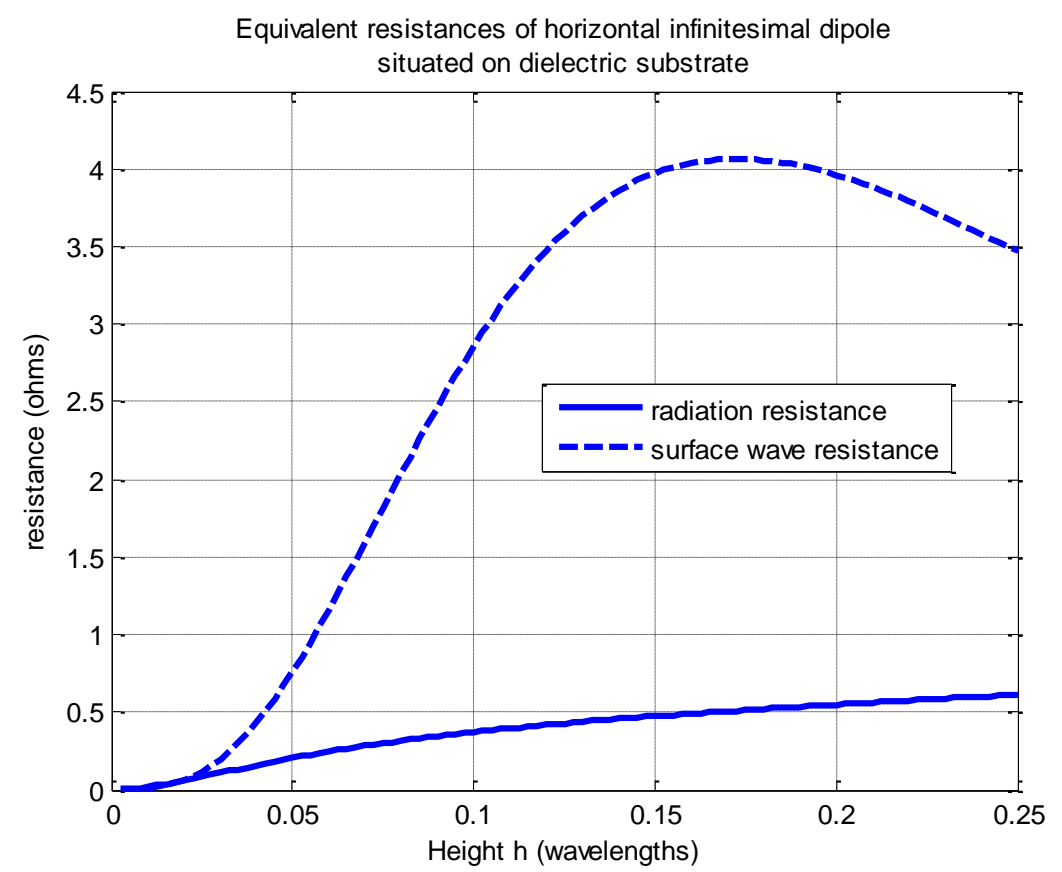

Fig. 11 Equivalent resistances of a horizontal infinitesimal dipole situated on a dielectric substrate; $\varepsilon_{r}=1.01 ; \mu_{r}=10 ; \ell=\lambda_{0} / 50$

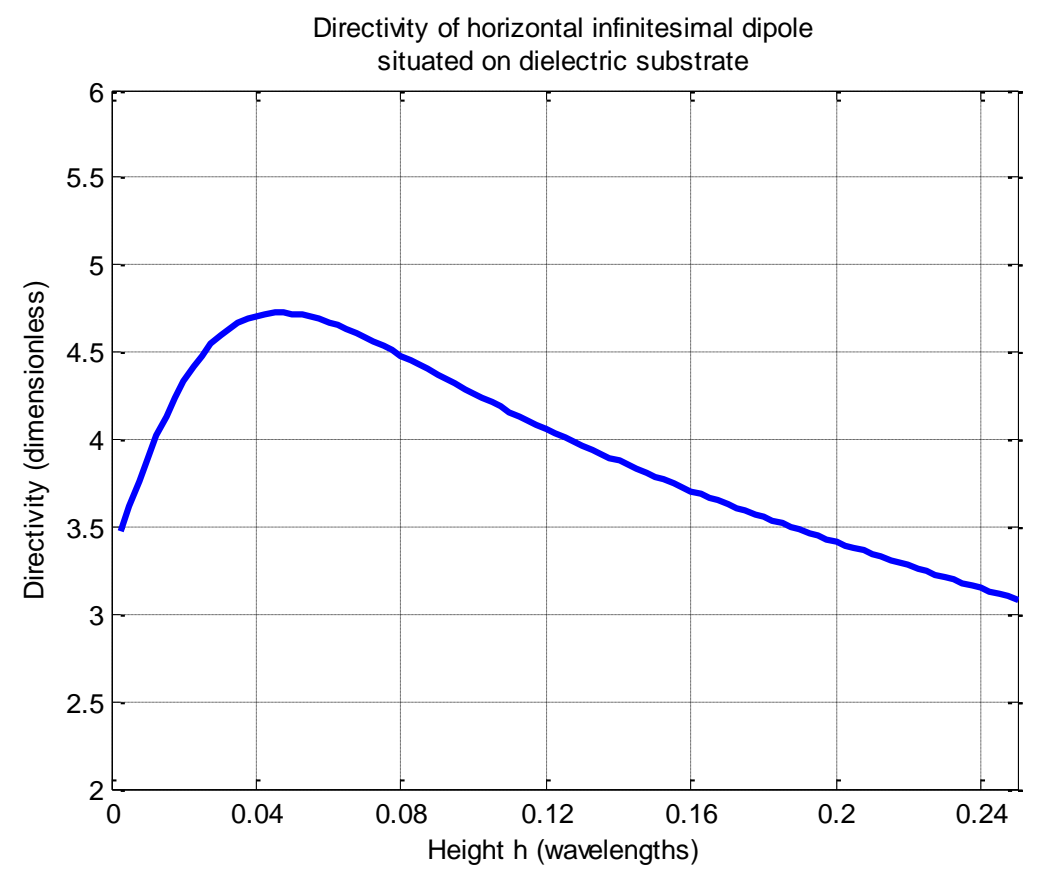

Fig. 12 Directivity of a horizontal infinitesimal dipole situated on a dielectric substrate; $\varepsilon_{r}=1.01 ; \mu_{r}=10 ; \ell=\lambda_{0} / 50$ 


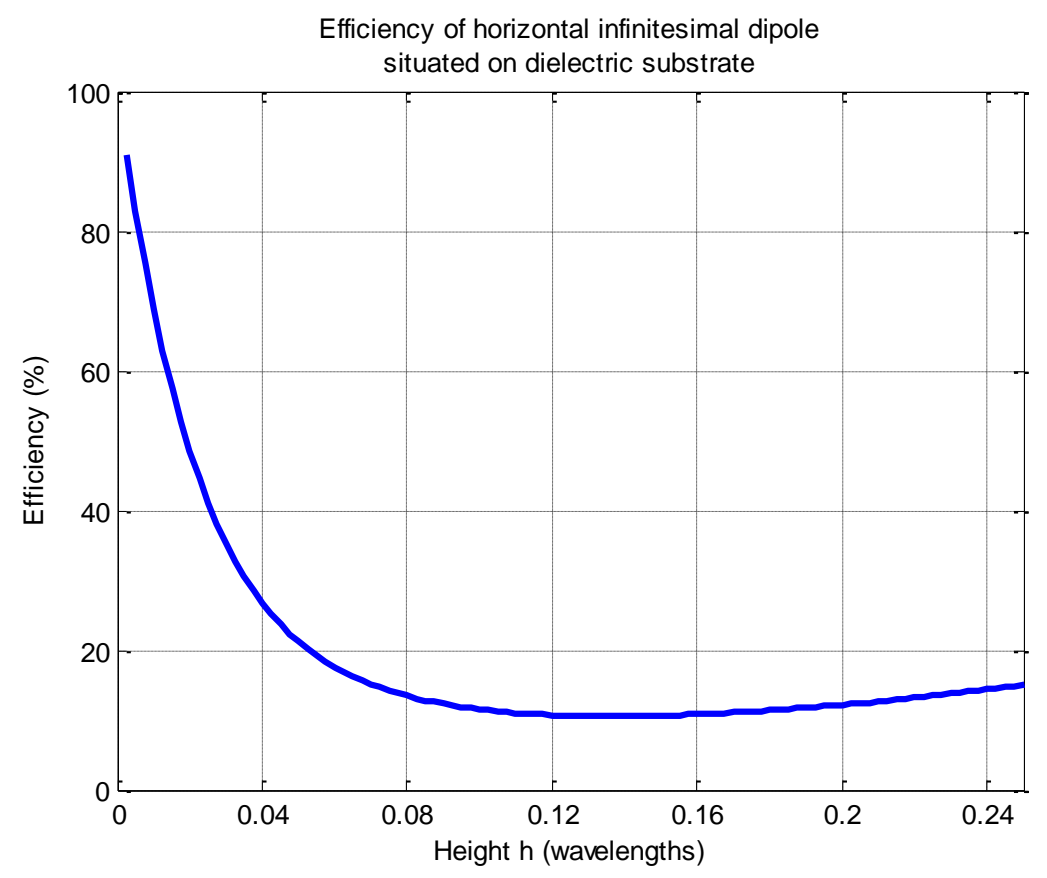

Fig. 13 Efficiency of a horizontal infinitesimal dipole situated on a dielectric substrate; $\varepsilon_{r}=1.01 ; \mu_{r}=10 ; \ell=\lambda_{0} / 50$

For $\varepsilon_{r}=10 ; \mu_{r}=10 ; \ell=\lambda_{0} / 50$

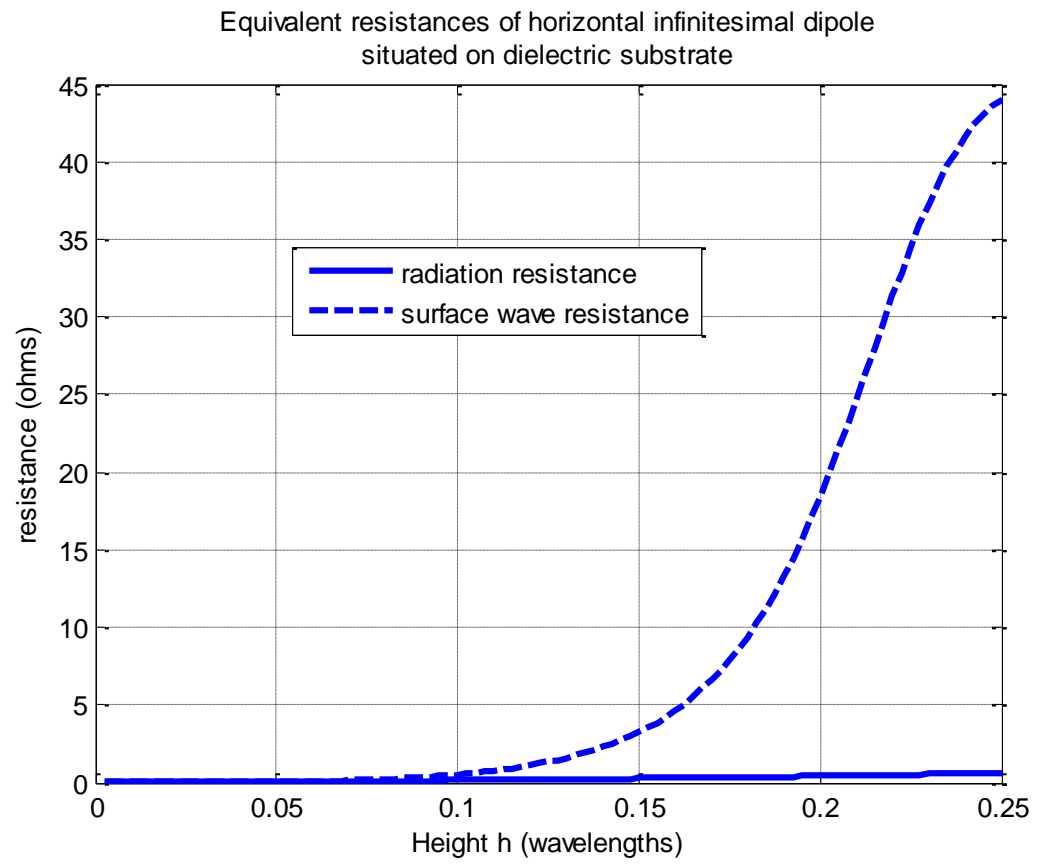

Fig. 14 Equivalent resistances of a horizontal infinitesimal dipole situated on a dielectric substrate; $\varepsilon_{r}=10 ; \mu_{r}=10 ; \ell=\lambda_{0} / 50$ 


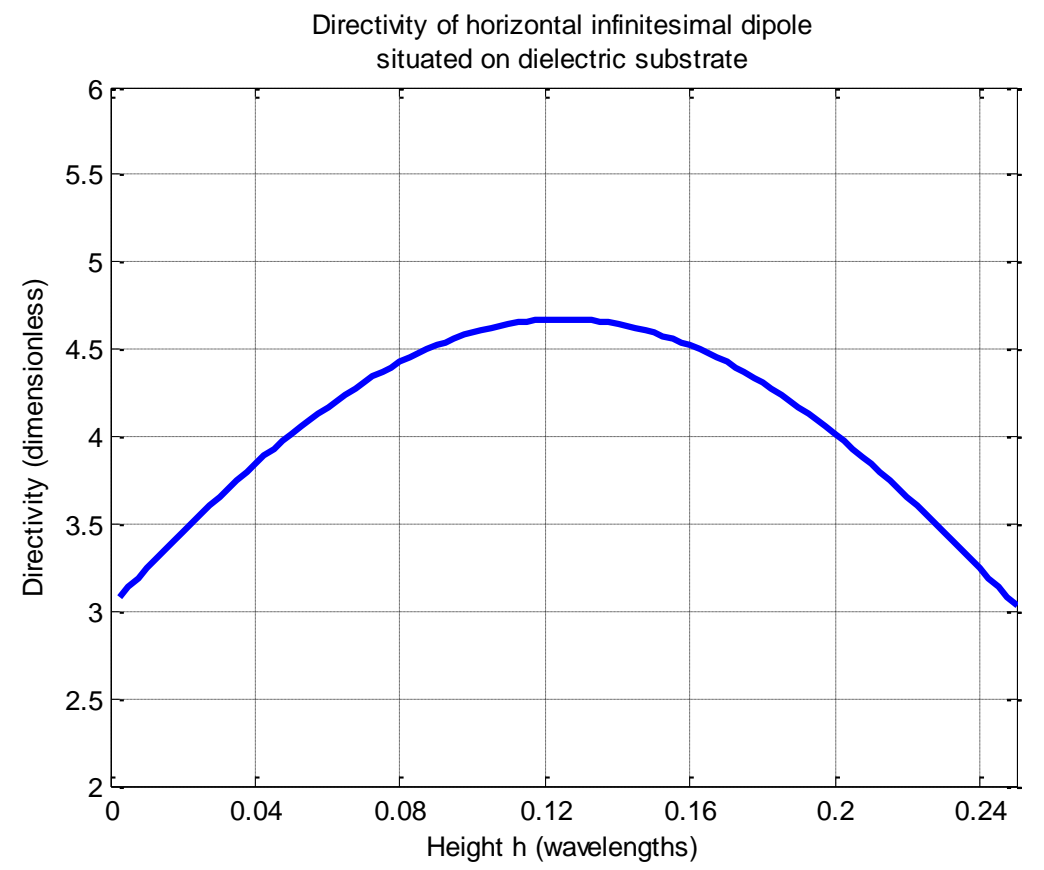

Fig. 15 Directivity of a horizontal infinitesimal dipole situated on a dielectric substrate; $\varepsilon_{r}=10 ; \mu_{r}=10 ; \ell=\lambda_{0} / 50$

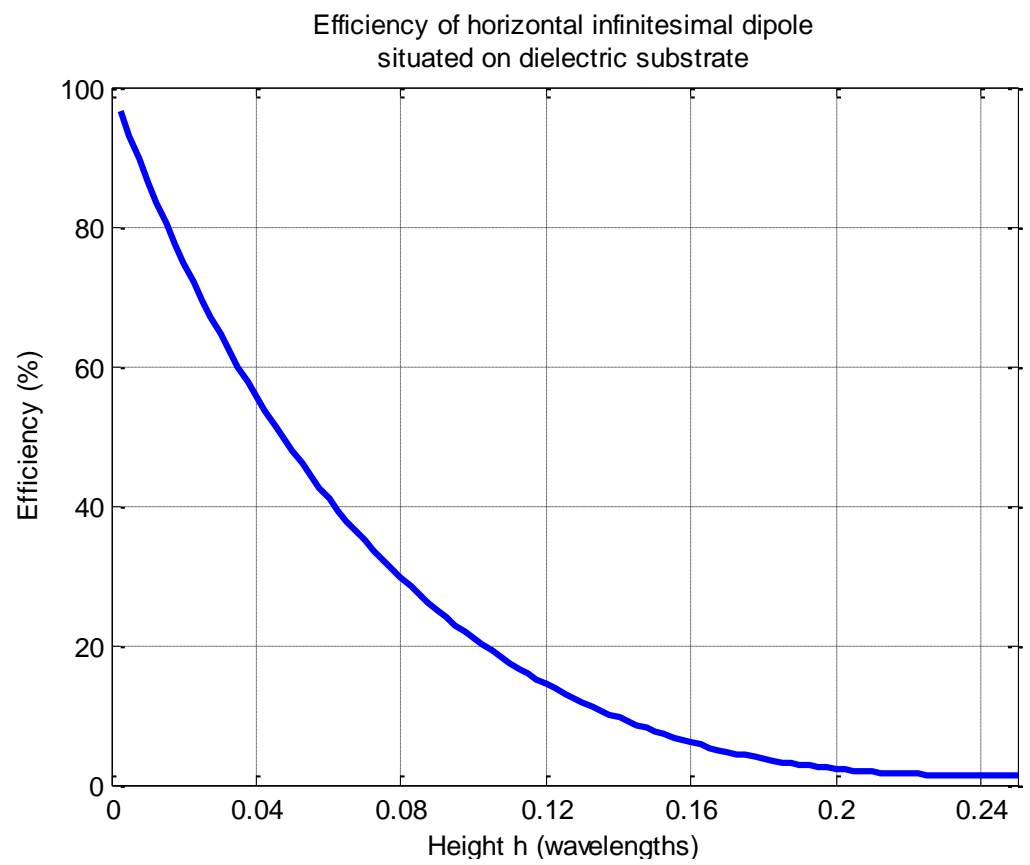

Fig. 16 Efficiency of a horizontal infinitesimal dipole situated on a dielectric substrate; $\varepsilon_{r}=10 ; \mu_{r}=10 ; \ell=\lambda_{0} / 50$ 


\section{Conclusion}

In this report, the solution of the boundary value problem associated with dipole radiation over grounded magnetodielectric substrates was treated. The mathematical details, which proved difficult to find in the literature, were reproduced. The analysis of these solutions in the subsequent sections derived the radiation field, surface wave fields, directivity, radiation resistance, and efficiency. Some numerical results were plotted in Figs. 5-16. From this small sampling of data, we can already conclude that the behavior of planar antennas printed on magnetodielectric substrates is very complicated and non-intuitive. For example, Fig. 14 indicates that a very small dipole printed on a very thin substrate could easily present an input impedance of $50 \mathrm{ohms}$ at its terminals. However, despite conventional wisdom that quarterwavelength substrates make very effective radiators, Fig. 14 indicates that essentially all of the available power is delivered to surface waves excited within the substrate itself, which will not necessarily lead to desirable performance.

Furthermore, a novel treatment of the complex valued square roots was shown to produce results in agreement with those discussed in the literature with an arguably less tortured mathematical procedure. Appendix B can be consulted for an explanation of this approach and a derivation of the properties of the complex valued square roots in the complex plane. This should find application to other boundary value problems (such as the present one) for which solutions are obtained using Fourier transforms and those that require the determination of inverse transforms to extract useful formulas. 


\section{References}

1. Sommerfeld A. Partial differential equations in physics. Academic Press. 1949.

2. Mosig JR, Gardiol FE. Analytical and numerical techniques in the Green's function treatment of microstrip antennas and scatterers. IEE Proceedings. March 1983:130(2).

3. Balanis CA. Antenna theory: analysis and design. $3^{\text {rd }}$ ed. Wiley. 2005.

4. Jackson DR. The spectral domain method in electromagnetic. short course notes, USNCURSI Radio Science Meeting. 2014.

5. Weiss SJ. Private communication. 2014. 
INTENTIONALLY LEFT BLANK. 
Appendix A. Boundary Conditions 
The boundary conditions used in the derivation of the magnetic vector potential are developed in this appendix. The preliminary assumption made in the analysis of the problem was that the electromagnetic field could be modeled by 2 components of the vector magnetic potential:

$$
\boldsymbol{A}=\widehat{\boldsymbol{x}} A_{x}+\widehat{\mathbf{z}} A_{z}
$$

The electromagnetic field associated with this vector potential is

$$
\begin{gathered}
\boldsymbol{H}=\frac{1}{\mu} \nabla \times \boldsymbol{A} \\
\boldsymbol{E}=\frac{1}{j \omega \varepsilon} \nabla \times \boldsymbol{H}
\end{gathered}
$$

Substituting Eq. A-1 into Eq. A-2 yields

$$
\begin{gathered}
\boldsymbol{H}=\frac{1}{\mu}\left[\widehat{\boldsymbol{x}} \frac{\partial A_{z}}{\partial y}+\widehat{\boldsymbol{y}}\left(\frac{\partial A_{x}}{\partial z}-\frac{\partial A_{z}}{\partial x}\right)-\hat{\boldsymbol{z}} \frac{\partial A_{x}}{\partial y}\right] \\
\boldsymbol{E}=\frac{1}{j \omega \mu \varepsilon}\left[\widehat{\boldsymbol{x}}\left(\frac{\partial^{2} A_{z}}{\partial x \partial z}-\frac{\partial^{2} A_{x}}{\partial z^{2}}-\frac{\partial^{2} A_{x}}{\partial y^{2}}\right)+\widehat{\boldsymbol{y}}\left(\frac{\partial^{2} A_{z}}{\partial y \partial z}+\frac{\partial^{2} A_{x}}{\partial x \partial y}\right)\right. \\
\left.+\hat{\boldsymbol{z}}\left(\frac{\partial^{2} A_{x}}{\partial x \partial z}-\frac{\partial^{2} A_{z}}{\partial x^{2}}-\frac{\partial^{2} A_{z}}{\partial y^{2}}\right)\right]
\end{gathered}
$$

Given the general form of solution, Eq. A-3 may be reformulated as follows:

$$
\begin{array}{r}
\widetilde{\boldsymbol{H}}=\frac{1}{\mu}\left[-\widehat{\boldsymbol{x}}\left(j k_{y} \widetilde{A_{z}}\right)+\widehat{\boldsymbol{y}}\left(\frac{\partial \widetilde{A_{x}}}{\partial z}+j k_{x} \widetilde{A_{z}}\right)+\widehat{\boldsymbol{z}}\left(j k_{y} \widetilde{A_{x}}\right)\right] \\
\widetilde{\boldsymbol{E}}=\frac{1}{j \omega \mu \varepsilon}\left[\widehat{\boldsymbol{x}}\left(-j k_{x} \frac{\partial \widetilde{A_{z}}}{\partial z}+\left(k^{2}-k_{x}^{2}\right) \widetilde{A_{x}}\right)-\widehat{\boldsymbol{y}}\left(j k_{y} \frac{\partial \widetilde{A_{z}}}{\partial z}+k_{x} k_{y} \widetilde{A_{x}}\right)\right. \\
\left.+\hat{\boldsymbol{z}}\left(-j k_{x} \frac{\partial \widetilde{A_{x}}}{\partial z}+\left(k^{2}-k_{z}{ }^{2}\right) \widetilde{A_{z}}\right)\right]
\end{array}
$$

$\mathrm{At}=-h$, a PEC interface requires

$$
\begin{aligned}
\hat{\boldsymbol{Z}} \times \widetilde{\boldsymbol{E}} & =0 \\
\hat{\boldsymbol{Z}} \cdot \widetilde{\boldsymbol{H}} & =0
\end{aligned}
$$

Substituting Eq. A-4 into Eq. A-7, we immediately obtain

$$
\left.\widetilde{\widetilde{A_{x_{2}}}}\right|_{z=-h}=0
$$

Then, if we apply Eq. A-6 to the $\widehat{\boldsymbol{y}}$ component of the electric field in Eq. A-5

$$
\left.\left(-j k_{y} \frac{\partial \widetilde{A_{z_{2}}}}{\partial z}-k_{x} k_{y} \widetilde{A_{x_{2}}}\right)\right|_{z=-h}=0
$$


Use of Eq. A-8 in Eq. A-9 yields the other boundary condition applicable at the PEC interface:

$$
\left.\frac{\partial \widetilde{A_{z_{2}}}}{\partial z}\right|_{z=-h}=0
$$

Now, we consider the boundary condition:

$$
\hat{\mathbf{z}} \times\left(H_{1}-H_{2}\right)=J_{s}
$$

In reality, there is no surface current, yet reconsider the current density:

$$
\boldsymbol{J}=\widehat{\boldsymbol{x}} \ell I_{0} \delta(\boldsymbol{r})=\widehat{\boldsymbol{x}} \ell I_{0} \delta(x) \delta(y) \delta(z) .
$$

Using the integral representation of the delta functions

$$
\begin{aligned}
& \delta(x)=\frac{1}{2 \pi} \int_{-\infty}^{\infty} e^{-j k_{x} x} d k_{x} \\
& \delta(y)=\frac{1}{2 \pi} \int_{-\infty}^{\infty} e^{-j k_{y} x} d k_{y}
\end{aligned}
$$

we have

$$
\boldsymbol{J}=\frac{1}{(2 \pi)^{2}} \int_{-\infty}^{\infty}\left[\widehat{\boldsymbol{x}} \ell I_{0} \delta(z)\right] e^{-j k_{x} x} e^{-j k_{y} y} d k_{x} d k_{y}
$$

We identify

$$
\tilde{\boldsymbol{J}}=\widehat{\boldsymbol{x}} \ell I_{0} \delta(z)=\widetilde{\boldsymbol{J}_{s}} \delta(z)
$$

and therefore

$$
\widehat{\boldsymbol{Z}} \times\left(\widetilde{\boldsymbol{H}_{1}}-\widetilde{\boldsymbol{H}_{2}}\right)=\widehat{\boldsymbol{x}} \ell I_{0}
$$

Using Eq. A-4 in Eq. A-16 yields 2 equations:

$$
\begin{gathered}
\qquad\left.\widetilde{A_{z_{1}}}\right|_{z=0^{+}}=\left.\frac{1}{\mu_{r}} \widetilde{A_{z_{2}}}\right|_{z=0^{-}} \\
\left.\left(\frac{\partial \widetilde{A_{x_{1}}}}{\partial z}-j k_{x} \widetilde{A_{z_{1}}}\right)\right|_{z=0}-\left.\frac{1}{\mu_{r}}\left(\frac{\partial \widetilde{A_{x_{2}}}}{\partial z}-j k_{x} \widetilde{A_{z_{2}}}\right)\right|_{z=0}=-\mu_{0} \ell I_{0}
\end{gathered}
$$

Rearranging Eq. A-18,

$$
\left.\left(\frac{\partial \widetilde{A_{x_{1}}}}{\partial z}-\frac{1}{\mu_{r}} \frac{\partial \widetilde{A_{x_{2}}}}{\partial z}\right)\right|_{z=0}-\left.j k_{x}\left(\widetilde{A_{z_{1}}}-\frac{1}{\mu_{r}} \widetilde{A_{z_{2}}}\right)\right|_{z=0}=-\mu_{0} \ell I_{0}
$$

Using Eq. A-17 in Eq. A-19, we obtain 


$$
\left.\frac{\partial \widetilde{A_{x_{1}}}}{\partial z}\right|_{z=0^{+}}-\left.\frac{1}{\mu_{r}} \frac{\partial \widetilde{A_{x_{2}}}}{\partial z}\right|_{z=0^{-}}=-\mu_{0} \ell I_{0}
$$

We can obtain another boundary condition on the vector potentials from

$$
\left.\widehat{\mathbf{z}} \cdot\left(\widetilde{\boldsymbol{H}_{1}}-\frac{1}{\mu_{r}} \widetilde{\boldsymbol{H}_{2}}\right)\right|_{z=0}=0
$$

which gives

$$
\left.\widetilde{A_{x_{1}}}\right|_{z=0^{+}}=\left.\widetilde{A_{x_{2}}}\right|_{z=0^{-}}
$$

Our final boundary condition must come from

$$
\hat{\boldsymbol{z}} \times\left.\left(\widetilde{\boldsymbol{E}}_{\mathbf{1}}-\widetilde{\boldsymbol{E}}_{\mathbf{2}}\right)\right|_{z=0}=0
$$

Applying Eq. A-23 to the $\widehat{\boldsymbol{y}}$ component of the electric field in Eq. A-5, we find

$$
\left.\left(-j k_{y} \frac{\partial \widetilde{A_{z_{1}}}}{\partial z}-k_{x} k_{y} \widetilde{A_{x_{1}}}\right)\right|_{z=0}-\left.\frac{1}{\mu_{r} \varepsilon_{r}}\left(-j k_{y} \frac{\partial \widetilde{A_{z_{2}}}}{\partial z}-k_{x} k_{y} \widetilde{A_{x_{2}}}\right)\right|_{z=0}=0
$$

Rearranging Eq. A-24,

$$
-\left.j k_{y}\left(\frac{\partial \widetilde{A_{z_{1}}}}{\partial z}-\frac{1}{\mu_{r} \varepsilon_{r}} \widetilde{A_{x_{1}}} \frac{\partial \widetilde{A_{z_{2}}}}{\partial z}\right)\right|_{z=0}-\left.k_{x} k_{y}\left(\widetilde{A_{x_{1}}}-\frac{1}{\mu_{r} \varepsilon_{r}} \widetilde{A_{x_{2}}}\right)\right|_{z=0}=0
$$

Substitution of Eq. A-22 into Eq. A-25 gives

$$
\left.\frac{\partial \widetilde{A_{z_{1}}}}{\partial z}\right|_{z=0^{+}}-\left.\frac{1}{\mu_{r} \varepsilon_{r}} \frac{\partial \widetilde{A_{z_{2}}}}{\partial z}\right|_{z=0^{-}}=\left.j k_{x} \frac{\mu_{r} \varepsilon_{r}-1}{\mu_{r} \varepsilon_{r}} \widetilde{A_{x_{1}}}\right|_{z=0}
$$

In total, there are 6 unique boundary conditions applicable to the magnetic vector potential. They have been derived in this appendix from the boundary conditions satisfied by the electric and magnetic fields. They are repeated together as Eq. A-27.

$$
\begin{gathered}
\left.\widetilde{A_{x_{2}}}\right|_{z=-h}=0 \\
\left.\frac{\partial \widetilde{A_{z_{2}}}}{\partial z}\right|_{z=-h}=0 \\
\left.\widetilde{A_{z_{1}}}\right|_{z=0^{+}}=\left.\frac{1}{\mu_{r}} \widetilde{A_{z_{2}}}\right|_{z=0^{-}} \\
\left.\frac{\partial \widetilde{A_{x_{1}}}}{\partial z}\right|_{z=0^{+}}-\left.\frac{1}{\mu_{r}} \frac{\partial \widetilde{A_{x_{2}}}}{\partial z}\right|_{z=0^{-}}=-\mu_{0} \ell I_{0} \\
\left.\widetilde{A_{x_{1}}}\right|_{z=0^{+}}=\left.\widetilde{A_{x_{2}}}\right|_{z=0^{-}} \\
\left.\frac{\partial \widetilde{A_{z_{1}}}}{\partial z}\right|_{z=0^{+}}-\left.\frac{1}{\mu_{r} \varepsilon_{r}} \frac{\partial \widetilde{A_{z_{2}}}}{\partial z}\right|_{z=0^{-}}=\left.j k_{x} \frac{\mu_{r} \varepsilon_{r}-1}{\mu_{r} \varepsilon_{r}} \widetilde{A_{x_{1}}}\right|_{z=0}
\end{gathered}
$$


Appendix B. Branch Cuts 
The definition of $k_{z_{1}}$ for real values of $k_{t}$ is

$$
\begin{gathered}
k_{z_{1}}=\sqrt{k_{0}^{2}-k_{t}^{2}} \quad, \quad k_{t}<k_{0} \\
k_{z_{1}}=-j \sqrt{{k_{t}{ }^{2}-k_{0}^{2}}^{2}} \quad, \quad k_{t}>k_{0}
\end{gathered}
$$

In order to employ the methods of complex integration, this definition must be extended to the whole complex $k_{t}$ plane. The definition we use for $k_{z_{1}}$ is

$$
k_{z_{1}} \equiv-\sqrt{\left|k_{0}{ }^{2}-k_{t}{ }^{2}\right|} \exp \left[\frac{j}{2} \arg _{0}\left(k_{0}{ }^{2}-k_{t}{ }^{2}\right)\right]
$$

In Eq. B-2, $\arg _{0}(z)$ is the value of $\arg (z)$ that lies in the interval $(0,2 \pi]$. This is depicted graphically as follows:

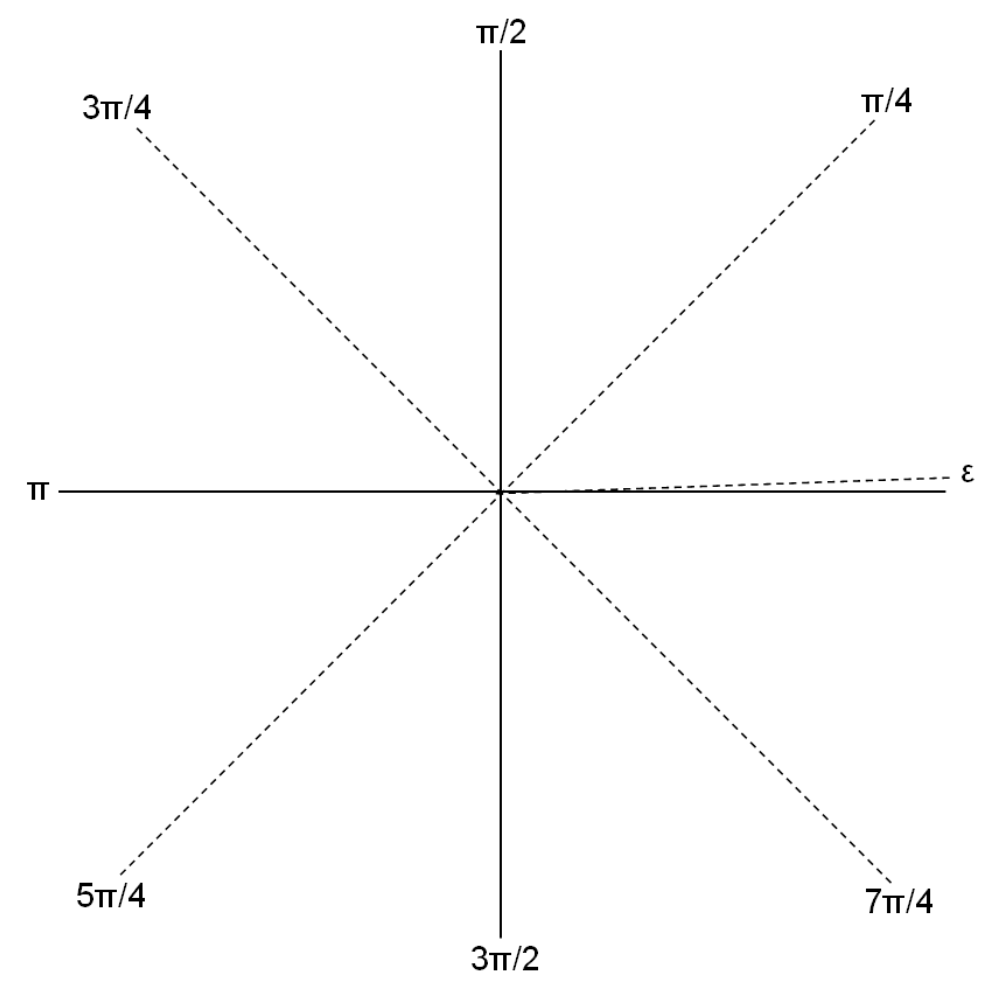

From the graph, we see that $\arg _{0}(z)$ is discontinuous if we cross the positive real axis in the $z$ plane. The real axis is said to form the branch cut for $\arg _{0}(z)$ and $\arg _{0}(z)$ is said to be a particular branch of the multi-valued $\arg (z)$ function. Now, $z=k_{0}{ }^{2}-k_{t}{ }^{2}$, so that we may map the branch cuts from the $z$ plane to the $k_{t}$ plane. The real and imaginary parts of $z, k_{0}, k_{t}$ are denoted as

$$
\begin{gathered}
z=x+j y \\
k_{0}=k_{0_{r}}+j k_{0_{i}} \\
k_{t}=k_{t_{r}}+j k_{t_{i}}
\end{gathered}
$$


Then,

$$
x+j y=\left({k_{0_{r}}}^{2}-{k_{0_{i}}}^{2}-k_{t_{r}}{ }^{2}+k_{t_{i}}{ }^{2}\right)+j 2\left(k_{0_{r}} k_{0_{i}}-k_{t_{r}} k_{t_{i}}\right)
$$

The positive real axis in the $z$ plane corresponds to the conditions

$$
\begin{gathered}
z=x+j y \\
x \geq 0 \\
y=0
\end{gathered}
$$

such that the equations defining the branch cuts in the $k_{t}$ plane are seen to be

$$
\begin{gathered}
k_{0_{r}}{ }^{2}-k_{0_{i}}{ }^{2}-k_{t_{r}}{ }^{2}+k_{t_{i}}{ }^{2} \geq 0 \\
k_{0_{r}} k_{0_{i}}-k_{t_{r}} k_{t_{i}}=0
\end{gathered}
$$

The second of these equations defines a hyperbola in the $k_{t}$ plane:

$$
k_{t_{r}}=\frac{k_{0_{r}} k_{0_{i}}}{k_{t_{i}}}
$$

Since $k_{0_{i}}$ must be a negative number to give attenuation, this hyperbola will appear as shown:

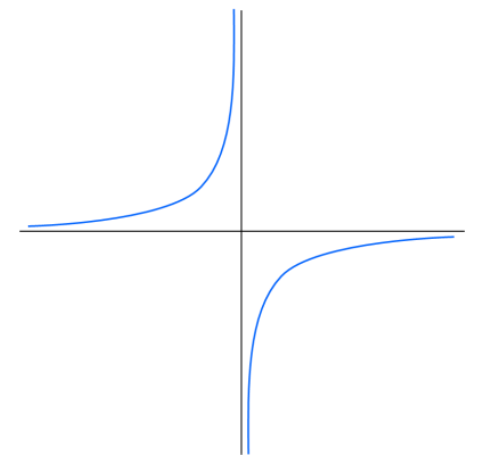

From Eq. B-6, the real part must be greater than 0. The endpoint of our hyperbolic curves must occur where $z=0$. This corresponds to the points $k_{t}= \pm k_{0}$. Our branch cuts are as follows:

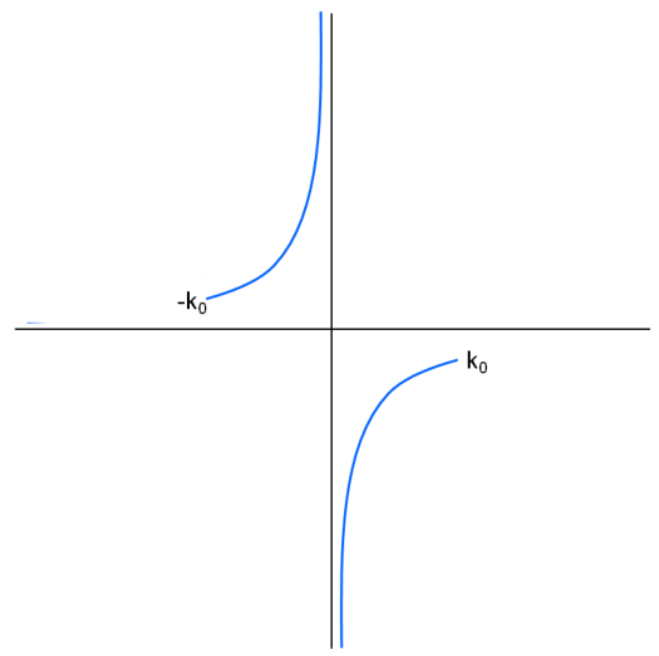


In the limit of no loss, $k_{0_{i}} \rightarrow 0$. Equation B-7 indicates that in this limit, the hyperbola will shrink inward to the coordinate axes. The governing equations for the branch cut reduce to

$$
\begin{gathered}
k_{0_{r}}{ }^{2}-k_{t_{r}}{ }^{2}+k_{t_{i}}{ }^{2} \geq 0 \\
k_{t_{r}} k_{t_{i}}=0
\end{gathered}
$$

These equations admit 2 solutions. That is,

$$
\begin{gathered}
k_{t_{r}}=0 \quad \text { (the imaginary axis) } \\
\left.k_{t_{i}}=0 ;\left|k_{t_{r}}\right| \leq\left|k_{0}\right| \quad \text { (the line segment on the real axis from }-k_{0} \text { to }+k_{0}\right)
\end{gathered}
$$

This is consistent with the physical picture of hyperbolas collapsing inward toward the coordinate axes. The branch cuts are depicted as follows:

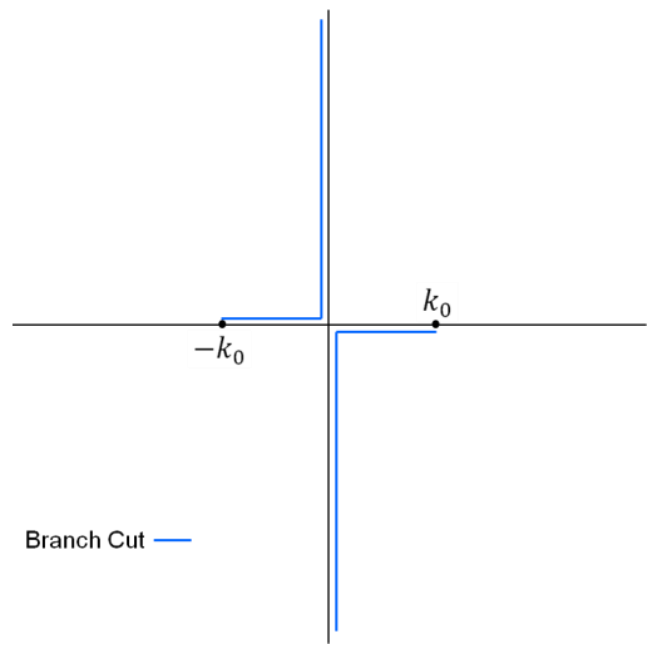

In view of Eq. B-1, and in order to obtain convergent integrals, we insist that $\operatorname{Im}\left(k_{z_{1}}\right) \leq 0$. We must verify that definition Eq. B-2 satisfies this requirement. In quadrant 1 , we know that $k_{t_{r}}>$ 0 and $k_{t_{i}}>0$. So that,

$$
\begin{gathered}
z=x+j y=\left(k_{0_{r}}{ }^{2}-k_{t_{r}}{ }^{2}+k_{t_{i}}{ }^{2}\right)-j 2 k_{t_{r}} k_{t_{i}} \\
y \leq 0
\end{gathered}
$$

Thus, in quadrant 1 , we have

$$
\frac{\pi}{2} \leq \frac{1}{2} \arg _{0}\left(k_{0}{ }^{2}-k_{t}{ }^{2}\right) \leq \pi
$$

and

$$
\begin{aligned}
& \operatorname{Re}\left\{\exp \left[\frac{j}{2} \arg _{0}\left(k_{0}{ }^{2}-k_{t}{ }^{2}\right)\right]\right\} \leq 0 \\
& \operatorname{Im}\left\{\exp \left[\frac{j}{2} \arg _{0}\left(k_{0}{ }^{2}-k_{t}{ }^{2}\right)\right]\right\} \geq 0
\end{aligned}
$$


In view of Eq. B-2 and Eq. B-12, we can conclude that within quadrant 1, the following inequalities hold:

$$
\begin{aligned}
& \operatorname{Re}\left(k_{z_{1}}\right) \geq 0 \\
& \operatorname{Im}\left(k_{z_{1}}\right) \leq 0
\end{aligned}
$$

This analysis can be repeated for quadrants $2,3,4$. The topology for $k_{z_{1}}$ is summarized as

\begin{tabular}{|c|c|}
\hline \multirow[t]{2}{*}{$\operatorname{Im}\left(k_{z_{1}}\right) \leq 0$} & $\begin{array}{l}\operatorname{Re}\left(k_{z_{1}}\right) \geq 0 \\
\operatorname{Im}\left(k_{z_{1}}\right) \leq 0\end{array}$ \\
\hline & $k_{0}$ \\
\hline$-k_{0}$ & \\
\hline $\operatorname{Re}\left(k_{z_{1}}\right) \geq 0$ & $\operatorname{Re}\left(k_{z_{1}}\right) \leq 0$ \\
\hline $\operatorname{Im}\left(k_{z_{1}}\right) \leq 0$ & $\operatorname{Im}\left(k_{z_{1}}\right) \leq 0$ \\
\hline Cut - & \\
\hline
\end{tabular}
follows: 


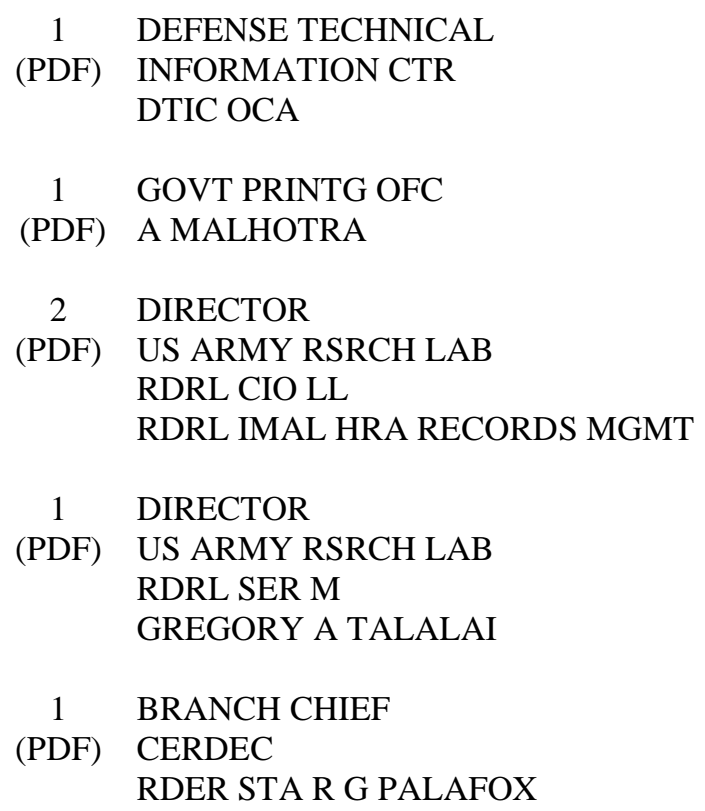

